\title{
Combined Use of Mathematical Optimization and Design of Experiments for the Maximization of Profit in a Four-Echelon Supply Chain
}

\author{
Daniel Arturo Olivares Vera, ${ }^{1}$ Elias Olivares-Benitez ${ }^{(\mathbb{D}},{ }^{2}$ Eleazar Puente Rivera, ${ }^{3}$ \\ Mónica López-Campos $\mathbb{D}^{4},{ }^{4}$ and Pablo A. Miranda $\mathbb{D}^{5}$ \\ ${ }^{1}$ Faculty of Information Technologies, Universidad de la Salle Bajío, León, GTO, Mexico \\ ${ }^{2}$ Facultad de Ingeniería, Universidad Panamericana, Zapopan, JAL, Mexico \\ ${ }^{3}$ Department of Engineering, Tecnologico de Monterrey, León, GTO, Mexico \\ ${ }^{4}$ Industrial Engineering Department, Universidad Técnica Federico Santa María, Valparaíso, Chile \\ ${ }^{5}$ School of Industrial Engineering, Pontificia Universidad Católica de Valparaíso, Valparaíso, Chile \\ Correspondence should be addressed to Mónica López-Campos; monica.lopezc@usm.cl
}

Received 5 October 2017; Revised 28 January 2018; Accepted 22 February 2018; Published 19 April 2018

Academic Editor: Borja Ponte

Copyright (C) 2018 Daniel Arturo Olivares Vera et al. This is an open access article distributed under the Creative Commons Attribution License, which permits unrestricted use, distribution, and reproduction in any medium, provided the original work is properly cited.

\begin{abstract}
This paper develops a location-allocation model to optimize a four-echelon supply chain network, addressing manufacturing and distribution centers location, supplier selection and flow allocation for raw materials from suppliers to manufacturers, and finished products for end customers, while searching for system profit maximization. A fractional-factorial design of experiments is performed to analyze the effects of capacity, quality, delivery time, and interest rate on profit and system performance. The model is formulated as a mixed-integer linear programming problem and solved by using well-known commercial software. The usage of factorial experiments combined with mathematical optimization is a novel approach to address supply chain network design problems. The application of the proposed model to a case study shows that this combination of techniques yields satisfying results in terms of both its behavior and the obtained managerial insights. An ANOVA analysis is executed to quantify the effects of each factor and their interactions. In the analyzed case study, the transportation cost is the most relevant cost component, and the most relevant opportunity for profit improvement is found in the factor of quality. The proposed combination of methods can be adapted to different problems and industries.
\end{abstract}

\section{Introduction}

The first aim of this research is to provide a mathematical model to optimize a supply chain network by maximizing the system profit, in which the performance of its solution is evaluated depending on changes observed in different levels of capacity, quality, delivery time, and the interest rate, four interrelated relevant aspects. The proposed model is applied to a supply chain that is composed of $r$ suppliers that deliver $m$ raw materials to $f$ manufacturers, who ship $p$ products to $d$ distribution centers, which finally send them to $c$ customers, as shown in Figure 1. The model optimizes the supply chain network yielding the highest benefit, taking into account demands and costs under a deterministic scenario, where all materials and products flowing through the supply chain can be represented by continuous amounts, and the optimization is made for a single time period. It is worthy to mention that optimization models can be solved using different techniques depending on the computational complexity of the model and the instance. The model proposed in this paper involves an NP-hard structure because of the decisions to locate operations at each level of the supply chain. The most direct approach is using commercial software that applies mathematical programming, which is limited to solving small or medium size instances for NP-hard problems. To solve larger instances, in many cases the use of metaheuristics like 


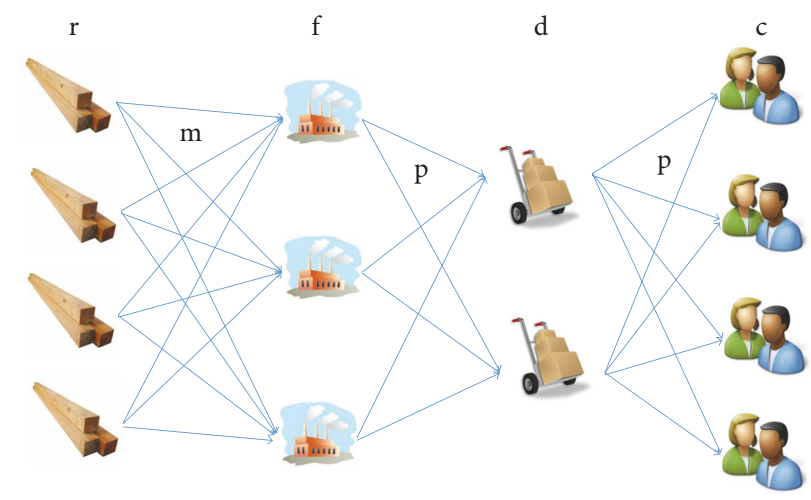

FIGURE 1: Structure of the analyzed supply chain (source: own elaboration).

the Black Hole Algorithm [1] or Evolutionary Algorithms [2] or others [3] is necessary.

One relevant novelty of this proposal is the integration of design of experiments (DOE) and optimization techniques, as a powerful quantitative tool to determine the best levels for the parameters and to quantify the significance and interaction of the involved factors, strengthening the analysis and managerial highlights. In some cases, the design of experiments can be especially useful, for the study of factors characterized by nonlinear functions or for the analysis of relationships in complex supply chain systems. To the best of our knowledge, there are few studies that combine DOE and optimization to solve supply chain design problems. Only, Bachlaus et al. [4] had an approximation to this issue. We found few studies of other kinds of supply chain problems solved by the combination of DOE and optimization, most of them dealing with the scheduling of transport routes, like in Raa [5], Hu et al. [6], and Zhang and Xu [7].

In our proposal, once the optimization model has been defined, we propose the use of the design of experiments to analyze the impact of each one of the studied parameters and their interactions, in the objective function. To this aim, an ANOVA analysis is done to analyze the effects of changes, which, unlike a traditional analysis of sensitivity, also takes into account the interactions between variables. Then, the significance of the main factors and interactions are extracted to give robust findings, as well as to find ideal values of the objective function, based on the knowledge of the behavior of the parameters. That is, the analyses fed back each other.

Each factor chosen to be analyzed in this study represents itself a whole area within organization management, but also these factors are indeed interrelated with each other. For example, an increase in supply chain capacity may reduce delivery times, while implying an economic cost, and at the same time, it may yield and increment in the complexity of the network design problem, by a combinatorial increment in the number of feasible solutions. In the case of supply chains, even manufacturing systems can be considered interrelated [8], generating very large and complex systems. Improvements in quality and delivery times require an economic investment, while low quality levels may entail the need to resort to external suppliers. This need for financial resources increases the relevance of the negotiated interest rate. Considering the aforementioned interrelations, one of the objectives of this research is to quantify their influence on the desired result, that is, the maximization of the net benefits. Design of Experiments is a well-known approach for doing these and other similar analyses.

The next subsections briefly discuss some of the most relevant factors for supply chain systems, integrating a review of some related literature. Subsequently, this document is composed of the problem description in Section 2, followed by the proposed mathematical model in Section 3. Afterwards, Section 4 focuses on the case study, where the effectiveness and usefulness of the proposed model are shown, and finally conclusions and future research lines are presented in Section 5.

1.1. Quality and Process Capability Index (Cp and Cpk) in the Supply Chain. In the literature, supply chain management and quality have been approached upon different perspectives, such as product quality and service quality [9-13], reprocessing and reworking in inventory models [14], quality costs [15], quality as a strategy [16-18], selection of suppliers [19], and the integration of capability indexes for the evaluation of products and performance of organizations [20-25].

For considering the quality factor in the supply chain, a well-known approach is specifying it regarding costs of quality (COQ) and cost of poor quality (COPQ) [15, 26]. In this study we will focus on the COPQ, based on the percentage of product that does not meet specifications which is estimated using the process capability index (Cpk), multiplied by the cost related to reprocessing and rework. Although other pertinent costs of poor quality of external or indirect nature may be considered [27], in this case the focus is on the costs derived from products out of specifications, since this is a chronic and important concern in the supply chain under study.

The process capability indexes $(C p$ or $C p k)$ define the capability of a process to meet customer specifications. Equation (1) represents the calculation of $C p$ using the upper specification limit (USL), the lower specification limit (LSL), and the standard deviation $(\sigma)$. Equation $(2 \mathrm{a})$ represents the calculation of the lower $C p k l$ using the mean $(\mu)$, the lower 
specification limit (LSL), and the standard deviation $(\sigma)$. Equation (2b) represents the calculation of the upper Cpku using the upper specification limit (USL), the mean, and the standard deviation.

$$
\begin{aligned}
C p & =\frac{\mathrm{USL}-\mathrm{LSL}}{6 \sigma} \\
C p k l & =\frac{\mu-\mathrm{LSL}}{3 \sigma} \\
C p k u & =\frac{\mathrm{USL}-\mu}{3 \sigma} .
\end{aligned}
$$

There is literature considering different approaches for using the indexes of process capability, such as graphs for analysis and comparison of $C p k l, C p k u$, and $C p$ [28], hypothesis tests for selection of suppliers [29], graphs relating capability index and price comparison [30], fuzzy integration of multiple criteria and attributes [31], simulation with self-correlated data [32], multivariate process capability [33], supplier selection decisions based on stochastic dominance [34], and supplier relationship analysis of manufacturing firms [35].

1.2. Delivery Time and Service Level. Improvements in production quality, reductions in defects and reprocessing, variability decrease, and operations synchronizing are recognized as key elements to achieve on-time delivery of raw material and finished products within the supply chain. The analysis of delivery performance is critical to manage and control the total time in a supply chain, since improvements in delivery times involve economic investment that must be quantified. Research has been carried out for these purposes, in which a time-of-delivery window and graph analysis are integrated to evaluate the performance of the deliveries, incorporating indexes of process capability as key elements in decision-making at the time of the purchase [36]. More recently, Bushuev [37] analyzes and demonstrates strategies for improving delivery performance when a supplier uses an optimally positioned delivery window to minimize the expected penalty costs. In other researches, dynamic mechanisms for early-warning safety are proposed for the quality of the supply chain where they operate according to the disturbance of waiting time [38].

1.3. Interest Rate. For the problem considered in this research, a desired or target quotation for the raw materials and the products through the supply chain is established. If the capacity of the suppliers that provide raw materials for a given price or a smaller one is reached, the supplier's quality performance does not allow accomplishing the contractual commitments, or the delivery standards are not adequate, then it is necessary to search for supply sources at higher costs, which must be financially supported through a loan. The conditions of these loans may vary according to their origin; therefore, it is necessary to consider a reference for the calculation of the amount to be paid on capital. In this paper, an interest rate is used as a benchmark. The interest rate is a representative fee of credit operations between banks, and it serves as a reference for loans to individuals. An infinite number of articles have been published regarding financial decisions and loans in the context of a supply chain. We highlight in this respect the recent work of Zhou et al. [39] who analyze how a capital-constrained retailer can determine their optimal advertising/ordering policy, selecting the best financing mode, according to their initial capital level and the interest rates of the financial services.

\section{Problem Statement}

The problem studied in this research is described in this section, which is based on the following assumptions:

(1) The use of data to calculate process capacities is regarded under stable process conditions and statistical control.

(2) The processes have a normal distribution behavior, and the individual quality for each process is independent of the others.

(3) The tolerance for the delivery time of raw material or products defined by the customer is called the "delivery time window."

(4) The interest rates are based on historical figures published from 2009 to 2015 by the country's Federal Bank.

The problem is to optimize the supply chain network in a static, single-period scenario, involving decisions of locating plants and distribution centers, supplier selection, quantities to be manufactured, and material flows between suppliers and manufacturers, from manufacturers to distribution centers, and between distribution centers and end customers, for a set of end products and raw materials. The aim is to maximize net benefits of the entire supply chain system, satisfying all end customer demands for each product.

2.1. Use of Capability Indexes in the Computation of Costs. The following paragraphs are aimed at describing and defining some of the main problem parameters, related to supply chain system costs.

The total quality cost is illustrated as CTC integrated by the sum of the poor quality costs of each of the RRTR, $R R T F$, and RRTD echelons for suppliers, manufacturers, and distribution centers, respectively. The calculation of the poor quality cost for the case of the suppliers that supply the material to the manufacturers, denominated as RRTR, is illustrated below. This same calculation will be made for the other RRTF and RRTD echelons.

We start calculating the upper processing capability index $C p k u$ and the lower processing capability index $C p k l$ of quality for both ends of the curve, for each of the materials and products that supply each of the suppliers, manufacturers, and distribution centers. Equation (3) represents $C p$ for the material $m$ sent by the supplier $r$ to the manufacturer $f$. This calculation is done by taking the mean, the lower specification limit, and the standard deviation. Equation (4a) represents the lower $C p k$ for the material $m$ sent by the supplier to the manufacturer $f$. This calculation is done using the mean, 
the lower specification limit, and the standard deviation. Equation (4b) represents the upper Cpk for the material $m$ provided by the supplier to the manufacturer $f$. This calculation is done by taking the upper specification limit, the mean, and the standard deviation.

$$
\begin{aligned}
C p C R_{m r f} & =\frac{\mathrm{USL}_{m r f}-\mathrm{LSL}_{m r f}}{6 \sigma_{m r f}} \\
C p k l C R_{m r f} & =\frac{\mu_{m r f}-\mathrm{LSL}_{m r f}}{3 \sigma_{m r f}} \\
C p k u C R_{m r f} & =\frac{\mathrm{USL}_{m r f}-\mu_{m r f}}{3 \sigma_{m r f}} .
\end{aligned}
$$

Calculated Cpk's will be used to determine the percentage of nonconforming material or product that does not meet established quality limits (see (5)). This will be done using the resulting probability density function for each concept, which results in the percentage of product that does not meet specifications (\% NC), taking into account the lower specification limit and the upper specification limit of the customer (LSL and USL) [24].

$$
\% \mathrm{NC}=1-\Phi\left(\frac{\mathrm{USL}-\mu}{\sigma}\right)+\Phi\left(\frac{\mu-\mathrm{LSL}}{\sigma}\right) .
$$

Once the percentage of nonconforming products that do not meet specifications is obtained, these are classified into two types: reprocessing and rework [40]. This percentage will be used to calculate the cost of poor quality taking into account the cost of reprocessing and the cost of rework, as well as the reprocessing rate and rework rate for each echelon.

(i) Reprocessing consists of processing a batch or subbatch of materials of unacceptable quality, by repeating the same process steps of a defined stage of production so that its quality can be made acceptable, that is, the repetition of one or more steps of a process during manufacturing after it is known that the product has not met the preset limits.

(ii) Rework consists of processing a batch or subbatch of materials of unacceptable quality by a process other than the one used to produce the material in the original way, to achieve acceptable quality [41], as shown in Figure 2.

For the calculation of the cost occasioned by the delivery time, a similar approach to the one of poor quality is considered. The capability indexes are calculated according to the maximum and minimum allowable delivery time, which acts as a specification limit (Linn, 2006). The mean and standard deviation are derived from the supplier's delivery records according to each echelon in the supply chain. These calculations are based on the concept of delivery time window proposed by Hsu et al. [36], as shown in Figure 3.

Once the indexes of capability for the delivery time have been obtained, the percentage of product that does not comply with the specification is calculated, either because it is delivered after the requested time or because it is delivered

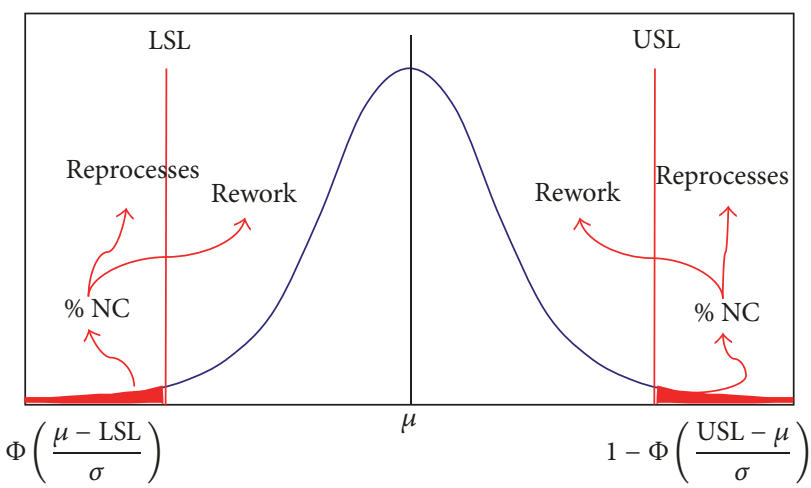

FIGURE 2: Percentage of nonconforming product for reprocessing and rework.

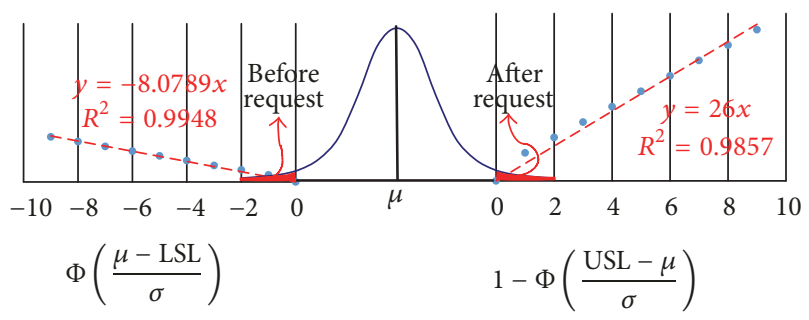

FIGURE 3: Cost of delivery time in the delivery time window.

before the requested time. The above will be used to fit a simple linear regression according to the days of lag with respect to the target time.

The cost of financing is integrated into the chain based on the manufacturer's price and the distribution center's price. The difference between the actual prices and the target price of the materials or products is considered, and this difference will be multiplied by the quantity sent and by the interest rate.

Once the costs of poor quality, delivery time, and financing have been calculated, the other costs are integrated into the model, consisting of the costs of operating and placing the product in the different facilities. These are the costs of purchasing material, the cost of producing, cost of transportation, and the cost of operating facilities [12, 42, 43]. It is important to mention that all other costs strongly depend on the general quality performance of the processes, especially the operation costs, which can be affected by a high rate of losses in production.

\section{Mathematical Formulation}

This section presents the indexes, parameters, decision variables, and the entire mathematical model. This is a mixedinteger linear program (MILP), having continuous variables representing the quantity of goods circulating in the supply chain and integer binary variables representing the decisions to open or not a location.

Indexes

$m$ : material

p: product 
$r$ : supplier

$f$ : manufacturer

$d$ : distribution center

c: customer

\section{Parameters}

\section{Quality Parameters}

$R R T R_{m r f}$ : total quality cost of material $m$ shipped from the supplier $r$ to manufacturer $f$ $R R T F_{p f d}$ : total quality cost of the product $p$ shipped from the manufacturer $f$ to the distribution center $d$

$R R T D_{p d c}$ : total quality cost of the product $p$ shipped from the distribution center $d$ to the customer $c$

$C p C R_{m r f}$ : quality value obtained from $C p$ for each supplier $r$

$C p C F_{p f d}$ : quality value obtained from $C p$ for each manufacturer $f$

$C p C D_{p d c}$ : quality value obtained from $C p$ for each distribution center $d$

$C p k C R_{m r f}$ : quality value obtained from $C p k$ for each supplier $r$

$C p k C F_{p f d}$ : quality value obtained from $C p k$ for each manufacturer $f$

$C p k C D_{p d c}$ : quality value obtained from $C p k$ for each distribution center $d$

$C p k u C R_{m r f}$ : quality value obtained from high Cpk for each supplier $r$

$C p k u C F_{p f d}$ : quality value obtained from high $C p k$ for each manufacturer $f$

$C p k u C D_{p d c}$ : quality value obtained from high $C p k$ for each distribution center $d$

$C p k l C R_{m r f}$ : quality value obtained from low $C p k$ for each supplier $r$

$C p k l C F_{p f d}$ : quality value obtained from $C p k$ low for each manufacturer $f$

$C p k l C D_{p d c}$ : quality value obtained from $C p k$ low for each distribution center $d$

$R R_{m r f}$ : reprocessing cost of the material $m$ of supplier $r$ to manufacturer $f$

$R T R_{m r f}$ : reworking cost of the material $m$ of supplier $r$ to manufacturer $f$

$T R P R_{m r f}$ : rate of reprocessing of material $m$ of supplier $r$ to manufacturer $f$

$T_{R T R_{m r f}}$ : rate of rework of material $m$ of supplier $r$ to manufacturer $f$

$R F_{p f d}$ : reprocessing cost of product $p$ of manufacturer $f$ to the distribution center $d$

$R T F_{p f d}$ : rework cost of product $p$ of manufacturer $f$ to the distribution center $d$

$T R P F_{p f d}$ : reprocessing rate of product $p$ of manufacturer $f$ to the distribution center $d$
$T R T F_{p f d}$ : reworking rate of product $p$ of manufacturer $f$ to the distribution center $d$

$R D_{p d c}$ : reprocessing cost of product $p$ of distribution center $d$ to the customer $c$

$R T D_{p d c}$ : rework cost of product $p$ of distribution center $d$ to the customer $c$

$T R P D_{p d c}$ : reprocessing rate of product $p$ of distribution center $d$ to customer $c$

$T R T D_{p d c}$ : reworking rate of product $p$ of distribution center $d$ to customer $c$

$\Phi R_{m r f}$ : probability density function of the quality characteristic of material $m$ from the supplier $r$ to manufacturer $f$

$\Phi F_{p f d}$ : probability density function of the quality characteristic of product $p$ from the manufacturer $f$ to the distribution center $d$

$\Phi D_{p d c}$ : probability density function of the quality characteristic of product $p$ from the distribution center $d$ to the customer $c$

\section{Delivery Time Parameters}

$C T E R_{m r f}$ : total cost per out-of-time delivery of material $m$ shipped from supplier $r$ to manufacturer $f$

$C T E F_{p f d}$ : total cost per out-of-time delivery of product $p$ shipped from manufacturer $f$ to the distribution center $d$

$C T E D_{p d c}$ : total cost per out-of-time delivery of product $p$ shipped from the distribution center $d$ to the customer $c$

TRUSL $_{m r f}$ : late delivery time limit of material $m$ delivered by supplier $r$ to manufacturer $f$

TRLSL $_{m r f}$ : earlier delivery time limit of material $m$ provided by supplier $r$ to manufacturer $f$

TFUSL $_{p f d}$ : late delivery time limit of product $p$ shipped by manufacturer $f$ to distribution center $d$

TFLSL $_{p r d}$ : early delivery time limit of product $p$ shipped by manufacturer $f$ to distribution center $d$

TDUSL $_{p d c}$ : late delivery time limit of product $p$ delivered by the distribution center $d$ to customer $c$

$T_{D L S L}{ }_{p d c}$ : early delivery time limit of product $p$ delivered by the distribution center $d$ to customer $c$

$T R \mu_{m r f}$ : average of delivery time of material $m$ provided by supplier $r$ to manufacturer $f$

$T R \sigma_{m r f}$ : standard deviation of delivery time of material $m$ provided by supplier $r$ to manufacturer $f$

$T F \mu_{p f d}$ : average of delivery time of product $p$ transported from the manufacturer $f$ to the distribution center $d$ 
$T F \sigma_{p f d}:$ standard deviation of delivery time of product $p$ transported from the manufacturer $f$ to the distribution center $d$

$T D \mu_{p d c}$ : average of delivery time of product $p$ delivered from the distribution center $d$ to customer $c$

$T D \sigma_{p d c}$ : standard deviation of delivery time of product $p$ delivered from the distribution center $d$ to the customer $c$

$P{ } R_{m r f}$ : constant of the slope of the equation of the cost of delivering after the limit time for the material $m$ of supplier $r$ to the manufacturer $f$

$D D T R_{m r f}$ : quantity of delivery days after the limit time of material $m$ from supplier $r$ to manufacturer $f$

PATR $_{m r f}$ : constant of the slope of the equation of the daily cost of delivering before the limit time of material $m$ from supplier $r$ to manufacturer $f$ $D A T R_{m r f}$ : quantity of delivery days before the limit time of material $m$ from supplier $r$ to manufacturer $f$

$P D T F_{p f d}$ : constant of the slope of the equation of the cost of delivering after the limit time for the product $p$ from manufacturer $f$ to distribution center $d$

$D D T F_{p f d}$ : quantity of delivery days after the limit time of product $p$ of manufacturer $f$ to distribution center $d$

PATF $_{p f d}$ : constant of the slope of the equation of the cost of delivering before the limit time for the product $p$ from manufacturer $f$ to distribution center $d$

$D A T F_{p f d}$ : quantity of delivery days before the limit time of product $p$ from the manufacturer $f$ to distribution center $d$

$P D T D_{p d c}$ : constant of the slope of the equation of the cost of delivering after the limit time for the product $p$ from the distribution center $d$ to customer $c$

$D D T D_{p d c}$ : quantity of delivery days after the limit time of product $p$ from the distribution center $d$ to customer $c$

$P A T D_{p d c}$ : constant of the slope of the equation of the cost of delivering before the limit time for the product $p$ from the distribution center $d$ to customer $c$

$D A T D_{p d c}$ : quantity of delivery days before the limit time of product $p$ from the distribution center $d$ to customer $c$

$T M D E R_{m r f}$ : rate of quantity delivered after the limit time of material $m$ from supplier $r$ to manufacturer $f$

$T M A E R_{m r f}$ : rate of material provided before the limit time, of material $m$ from supplier $r$ to manufacturer $f$
$T P D E F_{p f d}$ : rate of product delivered after the limit time, of product $p$ from manufacturer $f$ to distribution center $d$

$T P A E F_{p f d}$ : rate of product delivered before the limit time, of product $p$ from manufacturer $f$ to distribution center $d$

$T P D E D_{p d c}$ : rate of product delivered after the limit time of product $p$ from distribution center $d$ to customer $c$

$T P A E D_{p d c}$ : rate of product delivered before the limit time of product $p$ from distribution center $d$ to customer $c$

$\Phi T E R_{m r f}$ : probability density function of the delivery time of material $m$ from the supplier $r$ to manufacturer $f$

$\Phi T E F_{p f d}$ : probability density function of the delivery time of product $p$ from the manufacturer $f$ to distribution center $d$

$\Phi T E D_{p d c}$ : probability density function of the delivery time of product $p$ from the distribution center $d$ to customer $c$

\section{Interest Rate Parameters}

$C P R_{m r f}$ : cost of financing for a price higher than the target price of the material $m$ sent from the supplier $r$ to manufacturer $f$

$C P F_{p f d}$ : cost of financing for a price higher than the target price of product $p$ shipped from the manufacturer $f$ to distribution center $d$

$P R_{m r}$ : actual price of material $m$ from supplier $r$ $P F_{p f}$ : actual price of product $p$ from manufacturer $f$

$P D_{p d}$ : actual price of product $p$ from distribution center $d$

$T R_{m r}$ : target price of material $m$ of supplier $r$

$T F_{p f}$ : target price of product $p$ of manufacturer $f$

$F R F_{f}$ : interest rate for the manufacturer $f$

$F R D_{d}$ : interest rate for the distribution center $d$ $D Y_{r}$ : cost of starting operations with supplier $r$

$F F_{f}$ : cost of starting operations with manufacturer $f$

$D A_{d}$ : cost of starting operations of the distribution center $d$

$D O A_{f}$ : production capacity for the product $p$ by the manufacturer $f$

$D O V_{d}$ : delivery capacity for the product $p$ by the distribution center $d$

$D E M_{p c}$ : requested quantity of product $p$ by the customer $c$

$B V_{m r}$ : production capacity of material $m$ by supplier $r$

$M T_{m r f}$ : cost per unit to send material $m$ from supplier $r$ to manufacturer $f$ 
$A B_{p f d}$ : cost per unit to send product $p$ from manufacturer $f$ to distribution center $d$

$Y M_{p d c}$ : cost per unit to send product $p$ from the distribution center $d$ to customer $c$

$P O_{m p}$ : quantity of material $m$ to produce product $p$

$F A p_{f}$ : cost per unit to manufacture product $p$ made by manufacturer $f$

COSTMP1 $1_{m r f}$ : total cost of purchasing material $m$ to supplier $r$ by manufacturer $f$

\section{Decision Variables}

YPROV $_{r}$ : binary variable of "1" to start operations, or " 0 " not to start operations for the supplier $r$

$Y_{F A B R_{f}}$ : binary variable of "1" to start operations, or " 0 " not to start operations for the manufacturer $f$

$Y C D_{d}$ : binary variable of "1" to start operations, or " 0 " not to start operations for the distribution center $d$

$Q R_{m r f}$ : quantity of material $m$ from supplier $r$ to manufacturer $f$
$Q F_{p f d}:$ quantity of product $p$ from manufacturer $f$ to distribution center $d$

$Q D_{p d c}$ : quantity of product $p$ from the distribution center $d$ to customer $c$

$\operatorname{VOLMP} P_{m f}$ : volume received of material $m$ to the manufacturer $f$

$V O L F A B_{p f}$ : volume transformed of product $p$ by manufacturer $f$

Cost Modeling. The cost of poor quality is calculated by taking into account the cost of reprocessing $R R$ and the cost of rework $R T R$ as well as the reprocessing rate TRPR and the rework rate TRTR for each echelon. Here, it is important to mention that the calculation of reprocessing and rework costs is not trivial since, for example, a reworked defective part can be defective again and may not go directly back to the production flow. Equation (6) shows the calculations of rework to be performed, where $m$ represents the raw material, $r$ represents the supplier, and $f$ represents the manufacturer. Here, the quantity of material $m$ of supplier $r$ is multiplied by the percentage of product $p$ below the specification and the amount of product $p$ that is above the specification, which in turn are multiplied by the cost and rate of rework plus reprocessing cost and rate.

RRTR

$$
=\sum_{m=1}^{M} \sum_{r=1}^{R} \sum_{f=1}^{F}\left\{Q R_{m r f} \cdot\left[\left(\left(\Phi R_{m r f}\right) \cdot\left(-3 C p k l C R_{m r f}\right)\right)+\left(\left(\Phi R_{m r f}\right) \cdot\left(-3\left(2 C p C R_{m r f}-C p k u C R_{m r f}\right)\right)\right)\right] \cdot\left(R R_{m r f} \cdot T R P R_{m r f}+R T R_{m r f} \cdot T R T R_{m r f}\right)\right\} .
$$

In (7), it is possible to observe the calculation for the cost caused by deviations in the delivery time, which is affected by the days of lag with respect to the target time, as well as the quantity that was delivered out of time and the cost per day delivered out of time. In this first part, $Q R_{m r f}$ represents the quantity to be transported of material $m$ by the supplier $r$ to manufacturer $f$, multiplied by the rate $T M A E R_{m r f}$. This outcome is multiplied by the percentage delivered before the due date, which is finally multiplied by the product of the slope constant of the cost equation per day of delivery before the time limit $\left(P A T R_{m r f}\right)$ and the number of days of premature delivery of the material $m$ from the supplier $r$ to the manufacturer $f\left(D A T R_{m r f}\right)$.

$$
\begin{aligned}
& \text { CTER } \\
& =\sum_{m=1}^{M} \sum_{r=1}^{R} \sum_{f=1}^{F}\left\{\left[\left(R_{m r f} \cdot T M D E R_{m r f}\right)\left(1-\Phi T E R_{m r f}\left(\frac{T R U S L_{m r f}-T R \mu_{m r f}}{T R \sigma_{m r f}}\right)\right)\left(P D T R_{m r f} \cdot D D T R_{m r f}\right)\right]+\left[\left(R_{m r f} \cdot T M A E R_{m r f}\right)\left(\Phi T E R_{m r f}\left(\frac{T R \mu_{m r f}-T R L S L_{m r f}}{T R \sigma_{m r f}}\right)\right)\left(P A T R_{m r f} \cdot D A T R_{m r f}\right)\right]\right\} .
\end{aligned}
$$

The cost of the interest rate (CTP) is integrated into the model in (8) showing the total financing cost in the supply chain, based on the costs of the manufacturer $\left(C P R_{m r f}\right)$ and the distribution center $\left(C P F_{p f d}\right)$. Equation (9) shows the calculation for each echelon by means of multiplication only resulting from the difference of the real prices $\left(-P R_{m r}\right)$ and target price $\left(T R_{m r}\right)$ of the materials or products according to the case of the echelon that exceeds the target price for which the difference of the real price in relation to the target price will be calculated, and this difference will be multiplied by the amount sent $\left(Q R_{m r f}\right)$ and by the assigned interest rate.
This will be verified in three scenarios (low, average, and high rate) to determine the cost of the financing rate $\left(F R F_{f}\right)$, based on the value of the interest rate, calculated from the last five historical years recorded by the financial institution corresponding to the country.

$$
\begin{aligned}
& C T P=C P R_{m r f}+C P F_{p f d} \\
& C P R_{m r f} \\
& =\sum_{m=1}^{M} \sum_{r=1}^{R} \sum_{f=1}^{F}\left\{\max \left[0,\left(-P R_{m r}+T R_{m r}\right)\right] \cdot Q R_{m r f} \cdot F R F_{f}\right\} .
\end{aligned}
$$


The cost of purchasing material is obtained using the amount to be purchased for the actual price (COSTMP1) expressed in (10). The cost of producing (PRODUCE) in (11) is calculated for each product $p$ of each manufacturer $f$ by multiplying the cost of manufacturing by the volume manufactured. The cost of transportation (TRANSPORT) in (12) is the cost of sending the material or product to its requested destination along each of the echelons of the supply chain. The cost of operating (COSTOPERATE) in (13) of each of the echelons that make up the supply chain is represented by the multiplication of the cost of operating and its binary variable.

$$
\begin{aligned}
& \operatorname{COSTMP}_{m r f}=\sum_{m=1}^{M} \sum_{r=1}^{R}\left(Q R_{m r f} \cdot P R_{m r}\right) \\
& \text { PRODUCE }=\sum_{p=1}^{P} \sum_{f=1}^{F}\left(F A_{p f} \cdot V O L F A B_{p f}\right)
\end{aligned}
$$

$$
\begin{aligned}
& \text { TRANSPORT }=\sum_{m=1}^{M} \sum_{r=1}^{R} \sum_{f=1}^{F}\left(M T_{m r f} \cdot Q R_{m r f}\right) \\
& +\sum_{p=1}^{P} \sum_{f=1}^{F} \sum_{d=1}^{D}\left(A B_{p f d} \cdot Q F_{p f d}\right) \\
& +\sum_{p=1}^{P} \sum_{d=1}^{D} \sum_{c=1}^{C}\left(Y M_{p d c} \cdot Q D_{p d c}\right) \\
& \text { COSTOPERATE }=\sum_{r=1}^{R}\left(D Y_{r} \cdot Y P R O V_{r}\right)+\sum_{f=1}^{F}\left(F F_{f}\right. \\
& \cdot \text { YFABR } f)+\sum_{d=1}^{D}\left(D A_{d} \cdot Y C D_{d}\right) .
\end{aligned}
$$

Mixed-Integer Linear Program

Objective Function: Maximize Profitability

Profitability $=$ Earnings - Expenses

$$
\text { Earnings }=\sum_{p=1}^{P} \sum_{d=1}^{D}\left(Q D_{p d c} \cdot P D_{p d}\right)
$$

Expenses $=C T C+C T T+C T P+C O S T M P 1+P R O D U C E+T R A N S P O R T+C O S T O P E R A T E$

$$
\begin{gathered}
C T C=R R T R_{m r f}+R R T F_{p f d}+R R T D_{p d c} \\
C p C R_{m r f}=\frac{\mathrm{USL}_{m r f}-\mathrm{LSL}_{m r f}}{6 \sigma_{m r f}} \\
C p k u C R_{m r f}=\frac{\mathrm{USL}_{m r f}-\mu_{m r f}}{3 \sigma_{m r f}} \\
C p k l C R_{m r f}=\frac{\mu_{m r f}-\mathrm{LSL}_{m r f}}{3 \sigma_{m r f}}
\end{gathered}
$$

$$
C p k C R_{m r f}=\min \left\{C p k u C R_{m r f}, C p k l C R_{m r f}\right\}
$$

$$
\begin{aligned}
& R R T R_{m r f}=\sum_{m=1}^{M} \sum_{r=1}^{R} \sum_{f=1}^{F}\left\{Q R_{m r f} \cdot\left[\left(\Phi R_{m r f}\left(-3 C p k l C R_{m r f}\right)\right)+\left(\Phi R_{m r f}\left(-3\left(2 C p C R_{m r f}-C p k u C R_{m r f}\right)\right)\right)\right] \cdot\left[\left(R R_{m r f} \cdot T R P R_{m r f}\right)+\left(R T R_{m r f} \cdot T R T R_{m r f}\right)\right]\right\} \\
& C p C F_{p f d}=\frac{\mathrm{USL}_{p f d}-\mathrm{LSL}_{p f d}}{6 \sigma_{p f d}} \\
& C p k u C F_{p f d}=\frac{\mathrm{USL}_{p f d}-\mu_{p f d}}{3 \sigma_{p f d}} \\
& C p k l C F_{p f d}=\frac{\mu_{p f d}-\mathrm{LSL}_{p f d}}{3 \sigma_{p f d}} \\
& C p k C F_{p f d}=\min \left\{C p k u C F_{p f d}, C p k l C F_{p f d}\right\} \\
& R R T F_{p f d}=\sum_{p=1}^{P} \sum_{f=1}^{F} \sum_{d=1}^{D}\left\{Q F_{p f d} \cdot\left[\left(\Phi F_{p f d}\left(-3 C p k l C F_{p f d}\right)\right)+\left(\Phi F_{p f d}\left(-3\left(2 C p C F_{p f d}-C p k u C F_{p f d}\right)\right)\right)\right] \cdot\left[\left(R F_{p f d} \cdot T R P F_{p f d}\right)+\left(R T F_{p f d} \cdot T R T F_{p f d}\right)\right]\right\}
\end{aligned}
$$




$$
\begin{aligned}
& C p C D_{p d c}=\frac{\mathrm{USL}_{p d c}-\mathrm{LSL}_{p d c}}{6 \sigma_{p d c}} \\
& C p k u C D_{p d c}=\frac{\mathrm{USL}_{p d c}-\mu_{p d c}}{3 \sigma_{p d c}} \\
& C p k l C D_{p d c}=\frac{\mu_{p d c}-\mathrm{LSL}_{p d c}}{3 \sigma_{p d c}} \\
& C p k C D_{p d c}=\min \left\{C p k u C R_{p d c}, C p k l C R_{p d c}\right\} \\
& R R T D_{p d c}=\sum_{p=1}^{P} \sum_{d=1}^{D} \sum_{c=1}^{C}\left\{Q D_{p d c} \cdot\left[\left(\Phi D_{p d c}\left(-3 C p k l C D_{p d c}\right)\right)+\left(\Phi D_{p d c}\left(-3\left(2 C p C D_{p d c}-C p k u C D_{p d c}\right)\right)\right)\right] \cdot\left[\left(R D_{p d c} \cdot T R P D_{p d c}\right)+\left(R T D_{p d c} \cdot T R T D_{p d c}\right)\right]\right\} \\
& C T C=R R T R_{m r f}+R R T F_{p f d}+R R T D_{p d c} \\
& C T E R_{m f}=\sum_{m=1}^{M} \sum_{r=1}^{R} \sum_{f=1}^{F}\left\{\left[\left(Q_{m r f} \cdot T M D E R_{m r f}\right)\left(1-\Phi T E R_{m r f}\left(\frac{T R U S L_{m r f}-T R \mu_{m r f}}{T R \sigma_{m f}}\right)\right)\left(P D T R_{m r f} \cdot D D T R_{m r f}\right)\right]+\left[\left(Q R_{m f} \cdot T M A E R_{m r f}\right)\left(\Phi T E R_{m r f}\left(\frac{T R \mu_{m r f}-T R L S L_{m r f}}{T R \sigma_{m r f}}\right)\right)\left(P A T R_{m r f} \cdot D A T R_{m r f}\right)\right]\right\} \\
& C T E F_{p f d}=\sum_{p=1}^{P} \sum_{f=1}^{F} \sum_{d=1}^{D}\left\{\left[\left(Q F_{p f d} \cdot T P D E F_{p f d}\right)\left(1-\Phi T E F_{p f d}\left(\frac{T F U S L_{p f d}-T F \mu_{p f d}}{T F \sigma_{p f d}}\right)\right)\left(P D T F_{p f d} \cdot D D T F_{p f d}\right)\right]+\left[\left(Q F_{p f d} \cdot T P A E F_{p f d}\right)\left(\Phi T E F_{p f d}\left(\frac{T F \mu_{p f d}-T F L^{2} L_{p f d}}{T F \sigma_{p f d}}\right)\right)\left(P A T F_{p f d} \cdot D A T F_{p f d}\right)\right]\right\} \\
& C T E D_{p d c}=\sum_{p=1}^{p} \sum_{d=1}^{D} \sum_{c=1}^{C}\left\{\left[\left(Q D_{p d c} \cdot T P D E D_{p d c}\right)\left(1-\Phi T E D_{p d c}\left(\frac{T D U S L_{p d c}-T D \mu_{p d c}}{T D \sigma_{p d c}}\right)\right)\left(P D T D_{p d c} \cdot D D T D_{p d c}\right)\right]+\left[\left(Q D_{p d c} \cdot T P A E D_{p d c}\right)\left(\Phi T E D_{p d c}\left(\frac{T D \mu_{p d c}-T D L S L_{p d c}}{T D \sigma_{p d c}}\right)\right)\left(P A T D_{p d c} \cdot D A T D_{p d c}\right)\right]\right\} \\
& C T P=C P R_{m r f}+C P F_{p f d} \\
& C P R_{m r f}=\sum_{m=1}^{M} \sum_{r=1}^{R} \sum_{f=1}^{F}\left\{\max \left[0,\left(-P R_{m r}+T R_{m r}\right) \cdot Q R_{m r f} \cdot F R F_{f}\right]\right\} \\
& C P F_{p f d}=\sum_{p=1}^{P} \sum_{f=1}^{F} \sum_{d=1}^{D}\left\{\max \left[0,\left(-P F_{p f}+T F_{p f}\right) \cdot Q F_{p f d} \cdot F R D_{d}\right]\right\} \\
& \operatorname{COSTMP} 1_{m r f}=\sum_{m=1}^{M} \sum_{r=1}^{R}\left(Q R_{m r f} \cdot P R_{m r}\right) \\
& \text { PRODUCE }=\sum_{p=1}^{P} \sum_{f=1}^{F}\left(F A_{p f} \cdot V O L F A B_{p f}\right) \\
& \text { TRANSPORT }=\sum_{m=1}^{M} \sum_{r=1}^{R} \sum_{f=1}^{F}\left(M T_{m r f} \cdot Q R_{m r f}\right)+\sum_{p=1}^{P} \sum_{f=1}^{F} \sum_{d=1}^{D}\left(A B_{p f d} \cdot Q F_{p f d}\right)+\sum_{p=1}^{P} \sum_{d=1}^{D} \sum_{c=1}^{C}\left(Y M_{p d c} \cdot Q D_{p d c}\right) \\
& \text { COSTOPERATE }=\sum_{r=1}^{R}\left(D Y_{r} \cdot Y P R O V_{r}\right)+\sum_{f=1}^{F}\left(F F_{f} \cdot Y F A B R_{f}\right)+\sum_{d=1}^{D}\left(D A_{d} \cdot Y C D_{d}\right) \\
& \text { S.t. } \quad \sum_{d=1}^{D} Q D_{p d c} \geq D E M_{p c} \quad \forall p, c \\
& \sum_{d=1}^{D} Q F_{p f d} \leq V O L F A B_{p f} \quad \forall p, f \\
& \sum_{p=1}^{P} V O L F A B_{p f} \leq D O A_{f} \cdot Y F A B R_{f} \quad \forall f \\
& \sum_{p=1}^{P} \sum_{c=1}^{C} Q D_{p d c} \leq D O V_{d} \cdot Y C D_{d} \quad \forall d \\
& \sum_{p=1}^{P} \operatorname{VOLFAB}_{f p} \cdot P O_{m p} \leq \sum_{r=1}^{R} Q R_{m r f} \quad \forall m, f
\end{aligned}
$$




$$
\begin{gathered}
V O L M P_{m f}=\sum_{p=1}^{P} V O L F A B_{f p} \cdot P O_{m p} \quad \forall m, f \\
V O L M P_{m f}=\sum_{r=1}^{R} Q R_{m r f} \quad \forall m, f \\
\sum_{f=1}^{F} Q R_{m r f} \leq B V_{m r} \cdot Y P R O V_{r} \quad \forall m, r \\
\sum_{p=1}^{P} \sum_{f=1}^{F} Q F_{p f d} \leq D O V_{d} \cdot Y C D_{d} \quad \forall d \\
\sum_{f=1}^{F} Q F_{p f d}=\sum_{c=1}^{C} Q D_{p d c} \quad \forall p, d .
\end{gathered}
$$

The instance presented in this paper is not designed to explicitly reflect a trade-off between different kinds of costs. However, it is expected that the optimization model helps to compensate costs with others when obtaining the optimal solution. The objective is to maximize the profit, in (14), and is defined in (15a) as the difference between Earnings and Expenses. Equation (15b) is obtained by multiplying the quantity of product sold (QDpdc) by the actual price of the product (PDpd). Expenses are defined in (15c), integrated by the sum of the total costs of CTC, CTT, COSTMP1, PRODUCE, TRANSPORT, and COSTOPERATE. The total quality cost (CTC) is defined in (16) and it is comprised of the sum of the poor quality costs (reprocessing and rework) of $R R T R_{m r f}, R R T F_{p f d}$, and $R R T D_{p d c}$. Equation (17) determines the process capability index $(C p)$ for each supplier. Equation (19) calculates the low $C p k$ of the supplied material. Equation (20) determines the actual process capability index ( $C p k)$ for each supplier. Equation (21) is composed of the total cost of reprocessing and rework of each raw material $m$ that each supplier $r$ supplies to each manufacturer $f(R R T R)$. Equation (22) determines the process capability index $(C p)$ for each manufacturer. Equation (23) calculates the upper $C p k u$ of the manufacturer's product. Equation (24) calculates the lower $C p k l$. Equation (26) refers to the total cost of reprocessing and rework for each product $p$ by each manufacturer $f$. Equation (27) determines the process capability index $(C p)$ for each distribution center. Equation (28) calculates the upper Cpku of the products of the distribution center. Equation (29) calculates the lower $C p k l$ of the products of the distribution center. Equation (30) determines the actual process capability index $(C p k)$ for each distribution center. Equation (31) is composed of the total cost of reprocessing and reworking of each product $p$ that is distributed by the distribution center $d$ and that is supplied to each customer $c\left(R R T D_{p d c}\right)$. Equation (32) defines the total cost of delivery time (CTT) and it is comprised of the sum of the costs of $C T E R_{m r f}, C T E F_{p f d}$, and $C T E D_{p d c}$.

Equation (33) is composed of the total cost of deliveries before the permissible time requested and after the allowable time requested, for each raw material $m$ which each supplier $r$ supplies to each manufacturer $f\left(C T E R_{m r f}\right)$. This cost is taking into account the quantity multiplied by the historical rate of deliveries after the requested time, multiplied by the late arrival rate. Equation (34) is composed of the total cost of deliveries before the requested allowable time and after the permissible time of each product $p$ that is produced by each manufacturer $f$ and distributed by each distribution center $d$ $\left(C T E F_{p f d}\right)$ taking into account the quantity multiplied by the historical delivery rate after the requested time, multiplied by the late arrival rate. Equation (35) is composed of the total cost of deliveries before the allowable time requested and after the allowable time requested for each product $p$ that each distribution center $d$ supplies to each customer $c\left(C E T D_{p d c}\right)$. This cost is taking into account the quantity multiplied by the historical rate of deliveries after the requested time, multiplied by the late arrival rate. Equation (36) is composed of the total cost of financing (CTP) of manufacturers and distribution centers due to the difference of real prices and target prices $\left(C P R_{m r f}\right.$ and $\left.C P F_{p f d}\right)$. Equation (37) is composed of the financing cost $\left(C P R_{m r f}\right)$ for each manufacturer $f$ due to the difference of the actual price and the target price of each raw material $m$ which each supplier $r$ supplies, multiplied by the quantity and rate of the loan cost corresponding to each manufacturer $f$ for the amount requested. Equation (38) is composed of the cost of financing $\left(C P F_{p f d}\right)$ for each distribution center $d$ due to the difference of the actual price and the target price of each product $p$ that each manufacturer $f$ supplies, multiplied by the quantity and rate of the cost of loan corresponding to each distribution center $d$. Equation (39) defines the costs of buying the raw material $m$ of each supplier $r$ (COSTMP1). Equation (40) specifies the total cost of producing the products $p$ by the manufacturer $f$ (PRODUCE). Equation (41) represents the total costs of transportation from the supplier to the manufacturer, from the manufacturer to the distribution center, and from the distribution center to the customer, for each material $m$ and each product $p$ (TRANSPORT). Finally, (42) defines the total cost of operating provider, manufacturer, and distribution center with binary variables (COST OPERATE).

Constraints are defined by the following equations. Equation (43) ensures that the customer's demand is fulfilled. 
Equation (44) ensures that the quantity shipped per manufacturer based on the volume is respected. Equation (45) defines that the amount produced is less than the plant capacity. Equation (46) ensures that the quantity of the distribution center is less than that of those activated, using a binary variable to operate the warehouse. Equation (47) ensures that the quantity of material is less than the supplier capacity. Equation (48) converts volume of material to product. Equation (49) ensures that the amount of material is less than the quantity to be sent by the supplier. Equation (50) ensures that the quantity of material sent to the manufacturer is less than the available capacity of each supplier. Equation (51) defines a binary variable to ensure that the manufacturer operates taking into account capacity. Equation (52) ensures that the manufacturer's shipped quantity must be equal to the amount sent from the distribution center to the customer. Here, it is necessary to remember that the model is a single period, which will be appropriate to the planning interval required by the case.

\section{Case Study}

The supply chain analyzed in the paper consists of four echelons, with 40 suppliers $(r)$ supplying 30 materials $(m)$ to 30 manufacturers $(f)$, which fabricate and supply 20 end products $(p)$ to 30 distribution centers $(d)$, which send the end products to each of 40 customers $(c)$ to meet their demands $\left(D E M_{p c}\right)$.

We make changes in the values of capabilities, quality standards, delivery times, and interbank interest rate, each in three levels to be compared. The values of these parameters are generated randomly, following a normal distribution, with several instances according to the design of experiment that will be explained later.

Capacities were considered as follows: high capacity taking 55\% above total demand, average capacity taking 35\% above total demand, and, finally, a low capacity taking $25 \%$ above total demand. This procedure is performed for each supply chain echelon, that is, suppliers $\left(B V_{m r}\right)$, manufacturers $\left(D O A_{f}\right)$, and distribution centers $\left(D O V_{d}\right)$. The supply chain must meet customer demand $\left(D E M_{p c}\right)$.

For the quality factor, three levels are considered: high quality, medium quality, and low quality. The percentages of defects are determined according to the risks involved in each type of operation at each echelon, where the transformation stage yields higher portions of defects. Thus, the high quality considers a defect rate for suppliers $T D E F P V_{m r f}$ ranging from 0.00 to 0.03 , for manufacturers $T D E F F_{p f d}$ ranging from 0.00 to 0.05 , and for distribution centers $T D E F W_{p d c}$ ranging from 0.00 to 0.04 . The average quality considers a defect rate for suppliers $T D E F P V_{m r f}$ that ranges from 0.04 to 0.08 , for manufacturers $T D E F F_{p f d}$ ranging from 0.06 to 0.11 , and for distribution centers $T D E F W_{p d c}$ ranging from 0.05 to 0.09 . Low quality considers a defect rate for suppliers $T D E F P V_{m r f}$ ranging from 0.09 to 0.14 , for manufacturers $T D E F F_{p f d}$ ranging from 0.12 to 0.20 , and for distribution centers $T D E F W_{p d c}$ ranging from 0.10 to 0.16 as the minimum value.
For delivery time, different transport alternatives are considered, yielding three delivery times scenarios (low, medium, and high) for each of the supply chain echelons (manufacturer, distribution center, and customer):

(1) Low delivery time: if it is after the time of the requested date, this will be between 0 and 4 days, and if it is before the time of the requested date, this will be between 0 and 4 days.

(2) Average delivery time: if it is after the time of the requested date, this will be between 5 and 15 days, and if it is before the time of the requested date, this will be between 5 and 15 days.

(3) High delivery time: if it is after the time of the requested date, this will be between 16 and 30 days, and if it is before the time of the requested date, this will be between 16 and 30 days.

Finally, for determining the interest rate, three scenarios were considered taking into account the maximum, average, and minimum values recorded according to the periodicity in terms of 28, 91, and 182 days, issued by the country's Federal Bank, which reflect the conditions of the money market in national currency. The three calculated, maximum, average, and minimum, scenarios were used for each of two supply chain echelons (i.e., manufacturer and distribution center) as follows:

(1) Low interest rate, with a value of 3.3 percent, which will apply for those prices above the target value

(2) Average interest rate, with a value of 6 percent, which will be used for those prices above the target value

(3) High interest rate, with a value of 8.7 percent, which will apply for those prices above the target value.

4.1. Solving Procedure. In order to evaluate the impact of the different levels of capacity, quality, delivery time, and interest rate, a fractional-factorial $\left(2^{k-p}\right)$ experiment was performed where the following factors were considered: capacity (high, low), quality (high, low), delivery time (high, low), and interest rate (high, low). This results in a $2^{4-1}$ design, including central points for each of the concepts to explore a possible curvature in the model. Five instances were generated for each of the high and low combinations. Because of the fractional design, this accounts for 40 instances. Fifteen additional instances were generated for the central points included in the design, giving a total of 55 experimental runs.

The proposed model was solved for each instance of the case study using the GAMS-CPLEX software, on an Intel Core 3 processor, $3.0 \mathrm{~GB}$ RAM, $2.27 \mathrm{GHz}$ PC. The problem has 82,068 total variables, with 18,100 binary variables. It took between 17 and $25 \mathrm{~min}$ to solve each instance, giving a total of 982 minutes to obtain optimal solutions for all instances.

4.2. Results. Table 1 shows the average results of the nine scenarios of the design of fractional-factorial experiment $2^{4-1}$, with five central points and with five replicates, in which the calculated profits as well as the total cost and subcosts 


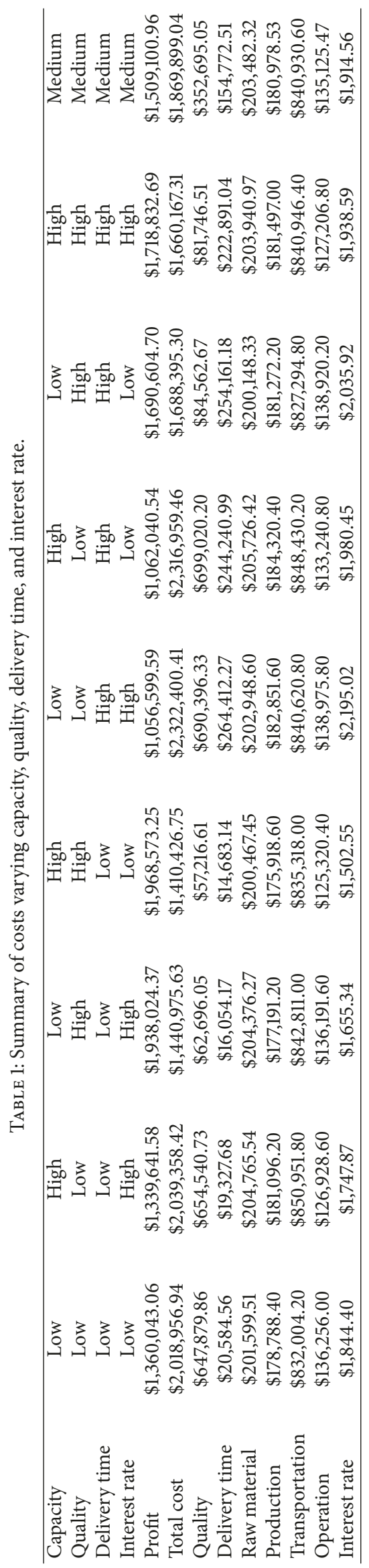


TABLE 2: Analysis of variance for profit of each scenario.

\begin{tabular}{|c|c|c|c|c|c|}
\hline Source & Sum of squares & $\mathrm{df}$ & Mean square & $F$ test & $P$ value \\
\hline A: capacity & $1.19992 E 9$ & 1 & $1.19992 E 9$ & 0.63 & 0.4313 \\
\hline B: quality & $3.8991 E 12$ & 1 & $3.8991 E 12$ & 2047.87 & 0.0000 \\
\hline C: delivery time & $7.26578 E 11$ & 1 & $7.26578 E 11$ & 381.61 & 0.0000 \\
\hline D: financing & $4.95733 E 8$ & 1 & $4.95733 E 8$ & 0.26 & 0.6123 \\
\hline $\mathrm{AB}+\mathrm{CD}$ & $3.39825 E 9$ & 1 & $3.39825 E 9$ & 1.78 & 0.1880 \\
\hline $\mathrm{AC}+\mathrm{BD}$ & $3.4579 E 8$ & 1 & $3.4579 E 8$ & 0.18 & 0.6719 \\
\hline $\mathrm{AD}+\mathrm{BC}$ & $4.39786 E 9$ & 1 & $4.39786 E 9$ & 2.31 & 0.1353 \\
\hline Total error & $7.7601 E 10$ & 47 & $1.90398 E 9$ & & \\
\hline Total (corr.) & $4.725 E 12$ & 54 & & & \\
\hline
\end{tabular}

$R$-squared $=98.1061$ percent; $R$-squared (adjusted by d.f.) $=97.824$ percent; standard error of est. $=43634.6$; average absolute error $=31174.2$; Durbin-Watson st. $=1.68847(P=0.1647)$; residual autocorrelation of Lag $1=0.149145$.

TABLE 3: Analysis of variance for profit (adjusted model).

\begin{tabular}{lcccc}
\hline Source & Sum of squares & $\mathrm{df}$ & Mean square & $F$ test \\
\hline B: quality & $3.8991 E 12$ & 1 & $3.8991 E 12$ & 2041.32 \\
C: delivery time & $7.26578 E 11$ & 1 & $7.26578 E 11$ & 380.39 \\
Total error & $9.93244 E 10$ & 52 & $1.91009 E 9$ & 0.0000 \\
Total (corr.) & $4.725 E 12$ & 54 & & 0.0000 \\
\hline
\end{tabular}

$R$-squared $=97.8979$ percent; $R$-squared (adjusted by d.f.) $=97.817$ percent; standard error of est. $=43704.5$; average absolute error $=35708.6$; Durbin-Watson st. $=1.72701(P=0.1321)$; residual autocorrelation of $\operatorname{Lag} 1=0.13133$.

TABLE 4: ANOVA for profit by scenario.

\begin{tabular}{lcccc}
\hline Source & Sum of squares & df & Mean square & $F$ test \\
\hline Between groups & $4.63616 E 12$ & 8 & $5.7952 E 11$ & 300.06 \\
Within groups & $8.88411 E 10$ & 46 & $1.93133 E 9$ & 0.0000 \\
Total (corr.) & $4.725 E 12$ & 54 & & \\
\hline
\end{tabular}

are expressed. The quality costs, delivery time costs, costs of raw material, costs of production, costs of transport, costs of operation, and cost of financing due to the interest rate are included, where the higher profit is fulfilled with a high capacity, high quality, low delivery time, and low interest rate.

The results of the Analysis of Variance (ANOVA) are shown in Table 2, where the significance of the main factors and double interactions can be observed, obtained with an adjusted $R^{2}$ of 98.3577 percent. Higher interactions cannot be scrutinized because of the fractional nature of the design. However, the most significant factors are the main factors: quality and delivery time. These results are confirmed visually in Figure 4.

The model is adjusted according to the significance, resulting in the main factors of the model shown in Table 3, with an adjusted $R^{2}$ of 97.817. In Figure 5, the adjusted Pareto chart for the significant effects is shown.

A Simple ANOVA was carried out to evaluate the contribution and variability of each one of the costs for each scenario within the fractional-factorial design, which are shown in Table 4 . It can be observed that there are significant differences for each one of the nine scenarios.

Thus, in Figure 6, it can be observed that the high capacity with the high quality (CP.H-C.H) is the group of scenarios with the best profit. The high quality with low capacity (CP.LC.H) is the second cheapest. These are closely followed by the combination of average capacity with average quality (CP.MC.M). Figure 7 shows the graph of averages of each scenario.

It can be observed that the highest profit is obtained with high capacity, high quality, low delivery time, and low financing (CP.H-C.H-T.L-F.L). However, the LSD test shows that there is no significant difference in the profit with low capacity, high quality, low delivery time, and high financing (CP.L-C.H-T.L-F.H), as can be seen in Table 5. Figure 7 shows the mean chart of each scenario.

Subsequently, an analysis in terms of costs is performed. These results are shown in Table 6, where it can be observed that there is a significant difference between the costs that affect the profit. In Figure 8, box-and-whisker plot of costs shows the variability of quality costs according to their levels.

A difference between the levels of quality and the dispersion of their data can be seen in Table 7. Also there, it can be observed that delivery time presents a dispersion in their data.

Table 8 presents the percentage of each cost related to the total cost, where transportation cost is the highest, followed by the quality cost and the cost of raw material acquisition. The behavior of profit and costs in an aggregated way is observed in Figure 9. 
TABLE 5: Multiple ranges testing for scenario profit.

(a) method: 95.0 percent LSD

\begin{tabular}{lccc}
\hline Level & Cases & Mean & Homogeneous groups \\
\hline CP.L-C.L-T.H-F.H & 5 & $1.0566 E 6$ & $\mathrm{X}$ \\
CP.H-C.L-T.H-F.L & 5 & $1.06204 E 6$ & $\mathrm{X}$ \\
CP.H-C.L-T.L-F.H & 5 & $1.33964 E 6$ & $\mathrm{X}$ \\
CP.L-C.L-T.L-F.L & 5 & $1.36004 E 6$ & $\mathrm{X}$ \\
CP.M-C.M-T.M-F.M & 15 & $1.5091 E 6$ & $\mathrm{X}$ \\
CP.L-C.H-T.H-F.L & 5 & $1.6906 E 6$ & $\mathrm{X}$ \\
CP.H-C.H-T.H-F.H & 5 & $1.71883 E 6$ & $\mathrm{X}$ \\
CP.L-C.H-T.L-F.H & 5 & $1.93802 E 6$ & $\mathrm{X}$ \\
CP.H-C.H-T.L-F.L & 5 & $1.96857 E 6$ & $\mathrm{X}$ \\
\hline
\end{tabular}

(b)

\begin{tabular}{|c|c|c|c|}
\hline Contrast & Sig. & Difference & $+/-$ limits \\
\hline CР.H-C.H-T.H-F.H, CP.H-C.H-T.L-F.L & * & -249741 & 55947.4 \\
\hline СР.Н-С.Н-Т.H-F.Н, CР.H-C.L-T.H-F.L & * & 656792 & 55947.4 \\
\hline CP.H-C.H-T.H-F.H, CP.H-C.L-T.L-F.H & * & 379191 & 55947.4 \\
\hline CP.H-C.H-T.H-F.H, CP.L-C.H-T.H-F.L & & 28228.0 & 55947.4 \\
\hline CР.H-C.H-T.H-F.H, CP.L-C.H-T.L-F.H & * & -219192 & 55947.4 \\
\hline CP.H-C.H-T.H-F.H, CP.L-C.L-T.H-F.H & * & 662233 & 55947.4 \\
\hline CP.H-C.H-T.H-F.H, CP.L-C.L-T.L-F.L & * & 358790 & 55947.4 \\
\hline СР.H-C.H-T.H-F.H, CР.M-С.M-T.M-F.M & * & 209732 & 45680.9 \\
\hline CP.H-C.H-T.L-F.L, CP.H-C.L-T.H-F.L & $*$ & 906533 & 55947.4 \\
\hline CP.H-C.H-T.L-F.L, CP.H-C.L-T.L-F.H & $*$ & 628932 & 55947.4 \\
\hline CP.H-C.H-T.L-F.L, CP.L-C.H-T.H-F.L & $*$ & 277969 & 55947.4 \\
\hline CP.H-C.H-T.L-F.L, CP.L-C.H-T.L-F.H & & 30548.9 & 55947.4 \\
\hline CP.H-C.H-T.L-F.L, CP.L-C.L-T.H-F.H & * & 911974 & 55947.4 \\
\hline CP.H-C.H-T.L-F.L, CP.L-C.L-T.L-F.L & * & 608530 & 55947.4 \\
\hline CP.H-C.H-T.L-F.L, CP.M-C.M-T.M-F.M & * & 459472 & 45680.9 \\
\hline CP.H-C.L-T.H-F.L, CP.H-C.L-T.L-F.H & * & -277601 & 55947.4 \\
\hline CP.H-C.L-T.H-F.L, CP.L-C.H-T.H-F.L & * & -628564 & 55947.4 \\
\hline CP.H-C.L-T.H-F.L, CP.L-C.H-T.L-F.H & * & -875984 & 55947.4 \\
\hline CP.H-C.L-T.H-F.L, CP.L-C.L-T.H-F.H & & 5440.96 & 55947.4 \\
\hline CP.H-C.L-T.H-F.L, CP.L-C.L-T.L-F.L & * & -298003 & 55947.4 \\
\hline CP.H-C.L-T.H-F.L, CP.M-C.M-T.M-F.M & * & -447060 & 45680.9 \\
\hline CP.H-C.L-T.L-F.H, CP.L-C.H-T.H-F.L & * & -350963 & 55947.4 \\
\hline CP.H-C.L-T.L-F.H, CP.L-C.H-T.L-F.H & * & -598383 & 55947.4 \\
\hline CP.H-C.L-T.L-F.H, CP.L-C.L-T.H-F.H & * & 283042 & 55947.4 \\
\hline CP.H-C.L-T.L-F.H, CP.L-C.L-T.L-F.L & & -20401.5 & 55947.4 \\
\hline CP.H-C.L-T.L-F.H, CP.M-C.M-T.M-F.M & * & -169459 & 45680.9 \\
\hline CP.L-C.H-T.H-F.L, CP.L-C.H-T.L-F.H & * & -247420 & 55947.4 \\
\hline CP.L-C.H-T.H-F.L, CP.L-C.L-T.H-F.H & * & 634005 & 55947.4 \\
\hline CP.L-C.H-T.H-F.L, CP.L-C.L-T.L-F.L & * & 330562 & 55947.4 \\
\hline CP.L-C.H-T.H-F.L, CP.M-C.M-T.M-F.M & * & 181504 & 45680.9 \\
\hline CP.L-C.H-T.L-F.H, CP.L-C.L-T.H-F.H & * & 881425 & 55947.4 \\
\hline CP.L-C.H-T.L-F.H, CP.L-C.L-T.L-F.L & * & 577981 & 55947.4 \\
\hline CP.L-C.H-T.L-F.H, CP.M-C.M-T.M-F.M & * & 428923 & 45680.9 \\
\hline CP.L-C.L-T.H-F.H, CP.L-C.L-T.L-F.L & * & -303443 & 55947.4 \\
\hline CP.L-C.L-T.H-F.H, CP.M-C.M-T.M-F.M & * & -452501 & 45680.9 \\
\hline CP.L-C.L-T.L-F.L, CP.M-C.M-T.M-F.M & * & -149058 & 45680.9 \\
\hline
\end{tabular}

$*$ indicates a significative difference. 


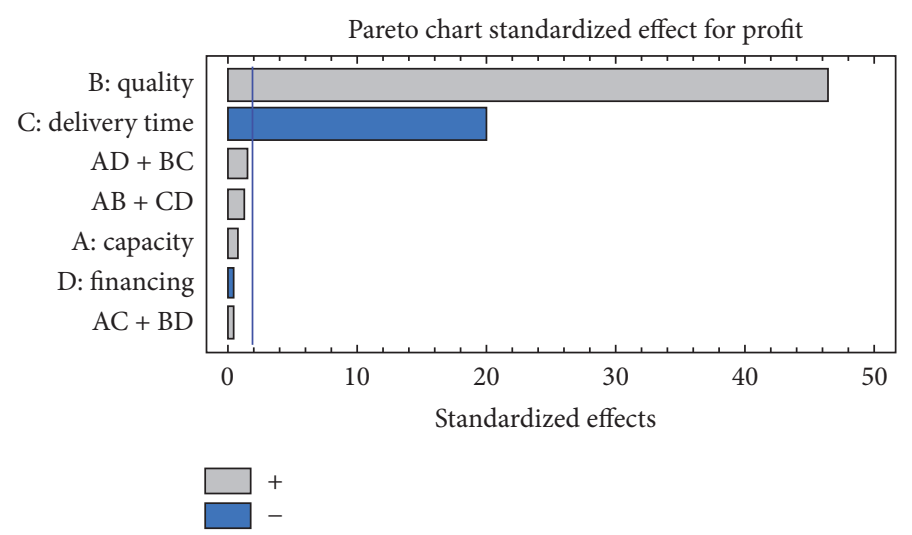

Figure 4: Standardized Pareto for the profit of factors.

TABLE 6: ANOVA cost per concept.

\begin{tabular}{|c|c|c|c|c|c|}
\hline Source & Sum of squares & $\mathrm{df}$ & Mean square & $F$ test & $P$ value \\
\hline Between groups & $2.50075 E 13$ & 6 & $4.16791 E 12$ & 375.18 & 0.0000 \\
\hline Within groups & 4.19929E12 & 378 & $1.11092 E 10$ & & \\
\hline Total (corr.) & $2.92067 E 13$ & 384 & & & \\
\hline
\end{tabular}

For Figures 10-17, the different combinations of factors are indicated in the $x$-axis (I.R. for interest rate, D.T. for delivery time, Q. for quality, and Cap. for capacity), and in the $y$-axis the disaggregated costs of quality, raw materials, production, transportation, operation, and financial are presented. Figure 10 shows the behavior of the profit for each scenario. Figure 11 shows the total costs, where it is observed how the high quality deeply reduces the total costs.

Figure 12 shows the behavior of the quality cost, which is the second highest of all costs. This figure also shows how, depending on the quality levels, these can significantly influence the profit. Figure 13 shows the costs of raw materials, where it is considered the third most important cost and one of the most stable.

Figure 14 shows the costs of production, which contribute between $7.9 \%$ and $12.5 \%$ of the costs. Figure 15 shows the behavior of transportation costs, which are the highest, ranging from $41.2 \%$ to $59.2 \%$ of total costs.

The operating costs are expressed in Figure 16 where they demonstrate being the lowest and the most stable since the operation of the plants is relatively constant. Finally, Figure 17 shows the lowest costs, which for the current rates do not impact the profit in an important way.

In Table 9, the number of facilities used in each echelon is presented for each scenario. Because of the differences in instances in each scenario, the numbers vary, such that only the minimum and maximum are presented for the group of instances of each scenario.

\subsection{Findings and Managerial Implications}

(i) In the studied case, the highest profit is fulfilled working with a high capacity, high quality, low delivery time, and low interest rate $(\$ 1,968,573)$. (ii) One of the effects of high capacity is that the number of facilities to operate can be reduced, having a better impact in profits due to a reduction in operating cost. However, an instance with high capacity may be harder to be solved since the number of potential combinations to locate facilities is larger.

(iii) As observed in the results (Table 9), capacity achieves the desired effect reducing the number of distribution centers needed to meet the demand. However, the increase in capacity does not affect the number of suppliers and plants. One explanation for this is that the full set of facilities is needed to be able to transform a large number of raw materials. Then, the supply chain is denser in interactions in the upstream echelons, and the density is reduced downstream, when the number of products is reduced, allowing a reduction in the number of distribution centers needed.

(iv) As expected, high quality impacts profit positively because a lower number of nonconforming products are generated. This not only reduces the costs of reprocessing and rework, but reduces the excess of material that must be considered to meet the demand and that must be purchased taking money from debt (interest rate).

(v) The previous insights agree with common sense, due to the double effect of having more capacity. If we have high capacity, the utility from sales is high too, and at the same time, fewer open facilities are needed. Nevertheless, using low capacity and high financing can give similar results in profit.

(vi) The difference observed between a high and a low quality level is not minor, since the quality has strong 
TABLE 7: Testing multiple ranges for costs.

(a) method: 95.0 percent LSD

\begin{tabular}{lccc}
\hline Concept & Cases & Mean & Homogeneous groups \\
\hline Financing & 55 & 1876.71 & $\mathrm{X}$ \\
Operation & 55 & 133492 & $\mathrm{X}$ \\
Delivery time & 55 & 138243 & $\mathrm{X}$ \\
Production & 55 & 180534 & $\mathrm{X}$ \\
Raw material & 55 & 203129 & $\mathrm{X}$ \\
Quality & 55 & 366922 & $\mathrm{X}$ \\
Transportation & 55 & 840106 & $\mathrm{X}$ \\
\hline
\end{tabular}

(b)

\begin{tabular}{lccc}
\hline Contrast & Sig. & Difference & + limits \\
\hline Quality, financing & $*$ & 365045 & 39520.0 \\
Quality, raw material & $*$ & 163793 & 39520.0 \\
Quality, operation & $*$ & 233430 & 39520.0 \\
Quality, production & $*$ & 186388 & 39520.0 \\
Quality, delivery time & $*$ & 228679 & 39520.0 \\
Quality, transportation & $*$ & -473184 & 39520.0 \\
Financing, raw material & $*$ & -201252 & 39520.0 \\
Financing, operation & $*$ & -131616 & 39520.0 \\
Financing, production & $*$ & -178657 & 39520.0 \\
Financing, delivery time & $*$ & -136366 & 39520.0 \\
Financing, transportation & $*$ & -838230 & 39520.0 \\
Raw material, operation & $*$ & 69636.7 & 39520.0 \\
Raw material, production & & 22595.4 & 39520.0 \\
Raw material, delivery time & $*$ & 64886.1 & 39520.0 \\
Raw material, transportation & $*$ & -636977 & 39520.0 \\
Operation, production & $*$ & -47041.3 & 39520.0 \\
Operation, delivery time & & -4750.54 & 39520.0 \\
Operation, transportation & $*$ & -706614 & 39520.0 \\
Production, delivery time & $*$ & 42290.8 & 39520.0 \\
Production, transportation & $*$ & -659573 & 39520.0 \\
Delivery time, transportation & & -701863 & 39520.0 \\
\hline
\end{tabular}

$*$ indicates a significant difference.

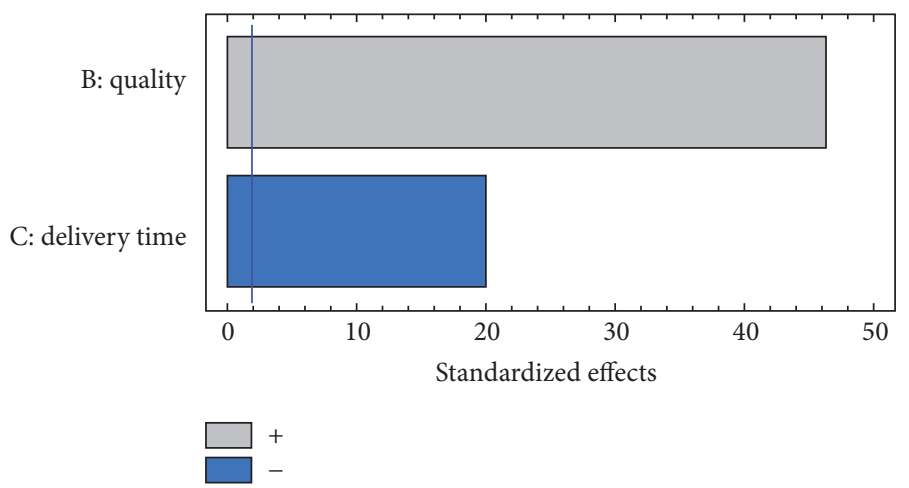

FIGURE 5: Graph of main effects for profit. 


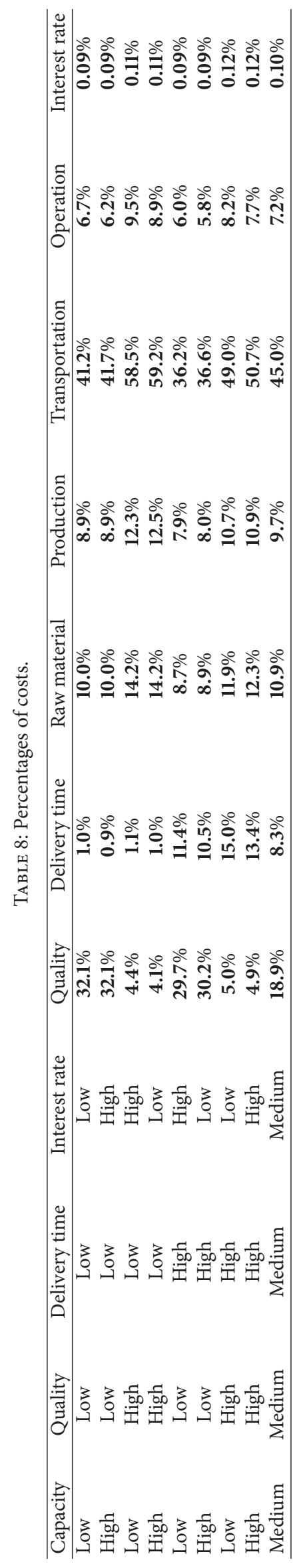


TABLE 9: Number of facilities used per echelon and scenario.

\begin{tabular}{|c|c|c|c|c|c|c|c|c|c|}
\hline \multirow[b]{2}{*}{ Capacity } & \multicolumn{3}{|c|}{ Instance } & \multicolumn{2}{|c|}{ Number of suppliers } & \multicolumn{2}{|c|}{ Number of plants } & \multicolumn{2}{|c|}{$\begin{array}{c}\text { Number of distribution } \\
\text { centers }\end{array}$} \\
\hline & Quality & Delivery & Interest rate & Minimum & Maximum & Minimum & Maximum & Minimum & Maximum \\
\hline High & High & High & High & 40 & 40 & 30 & 30 & 23 & 29 \\
\hline High & High & Low & Low & 37 & 40 & 30 & 30 & 21 & 24 \\
\hline High & Low & High & Low & 40 & 40 & 29 & 30 & 24 & 28 \\
\hline High & Low & Low & High & 37 & 40 & 30 & 30 & 22 & 29 \\
\hline Low & High & High & Low & 40 & 40 & 30 & 30 & 27 & 30 \\
\hline Low & High & Low & High & 40 & 40 & 30 & 30 & 26 & 28 \\
\hline Low & Low & High & High & 40 & 40 & 30 & 30 & 27 & 30 \\
\hline Low & Low & Low & Low & 40 & 40 & 30 & 30 & 24 & 30 \\
\hline Medium & Medium & Medium & Medium & 40 & 40 & 30 & 30 & 23 & 30 \\
\hline
\end{tabular}

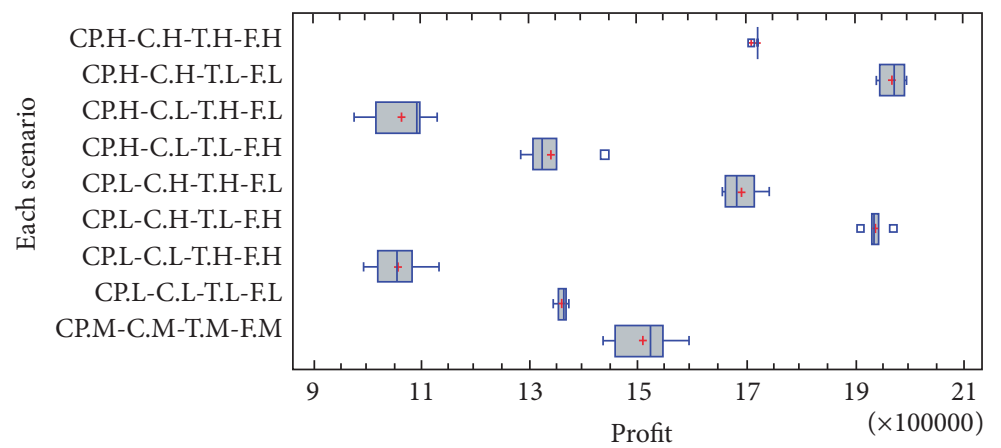

FIGURE 6: Box-and-whisker plot of profit.

relationships with all the rest of the factors, and a domino effect is generally observed when quality is improved. From this, we can argue that the quality performance can be even more significant than some logistics costs in reaching an adequate profit.

(vii) In this case, regarding costs, transportation yields the highest impact, followed by quality and then the cost of raw material. This is especially true in plants with operations relatively unchanging.

(viii) According to what has been observed, the two factors that most influence the value of the objective function are firstly the quality and then the delivery time. In fact, the quality factor has a little more than double the influence over profit than the delivery time. In this specific instance, the interactions between factors are not significant.

(ix) The exposed analysis has been done for a single time period. This time period can be as short or as long as the consideration of the demand and also according to the nature of the location decisions, which are usually long-term.

\section{Conclusions}

This paper proposes and studies a network design problem for a four-level supply chain (suppliers, plants, distribution centers, and end customers), where decisions include opening facilities (plants and distribution centers), supplier selection, and flows between the existing echelons. The problem is formulated as a mixed-integer linear programming model that maximizes the system profit depending on the sales price of the end products and the underlying system costs. The latter involve costs of operating facilities, transportation, raw material acquisition, and production activities. Additionally, a main contribution of this work is adding to the model the quality, delivery time, and financing aspects. The model maximizes the profit of the system in order to meet customer demand for finished products.

One primary and novel contribution of this work is the statistical analysis by designing experiments related to the impact on the system profit caused by changes in the levels of capacity, quality, delivery time, and interest rate for each of the actors involved in the supply chain. The results of the statistical analysis show that the transport cost component contributes most to the total cost, affecting the profit. However, regarding variability, the most important factor is 


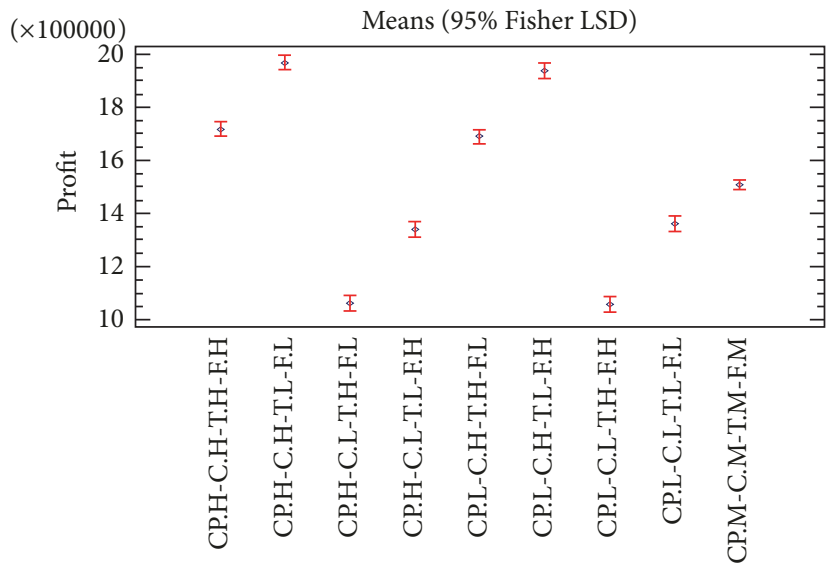

Each Scenario

FIgUre 7: Means graph.

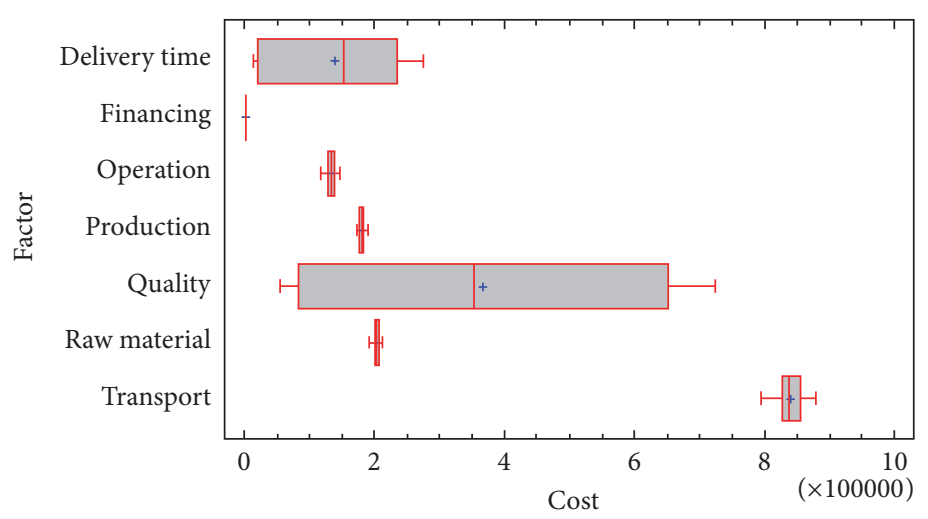

FIGURE 8: Box-and-whisker plot of costs.

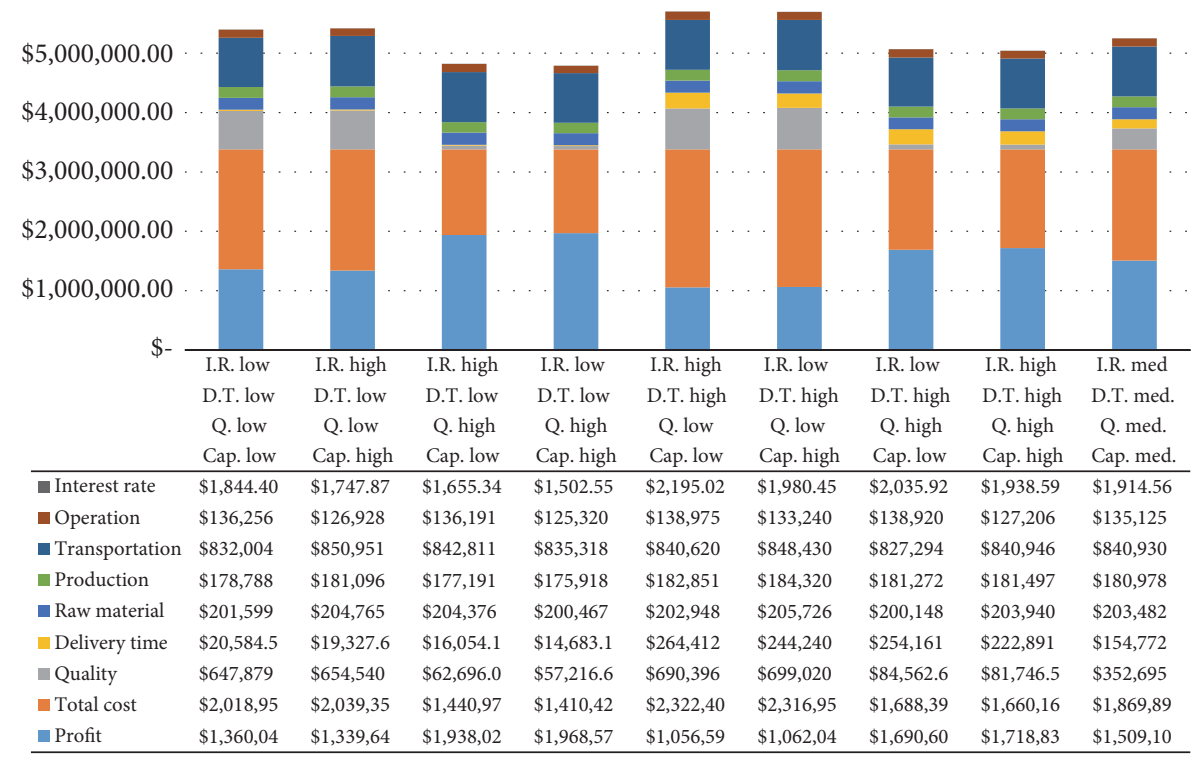

Figure 9: Profit, total costs, and subcosts. 


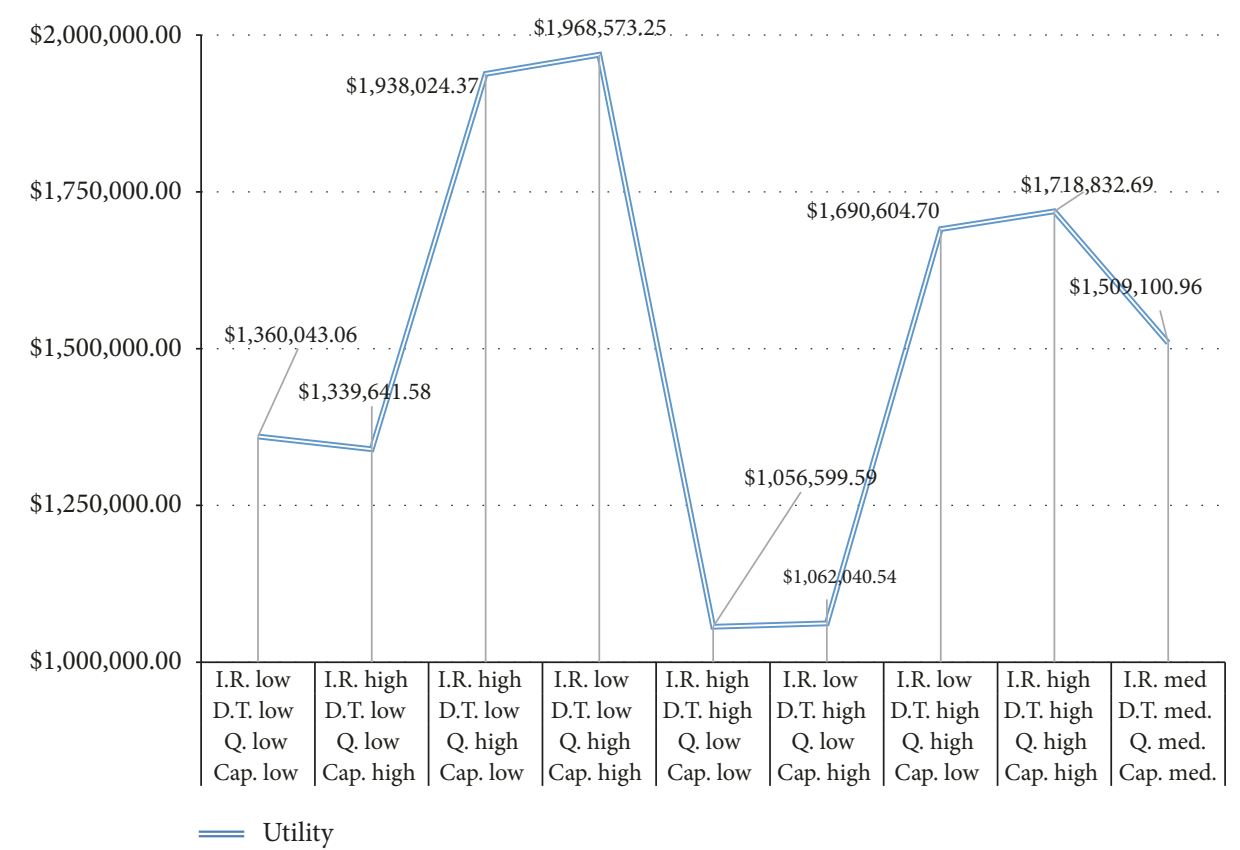

FIgUre 10: Total profit.

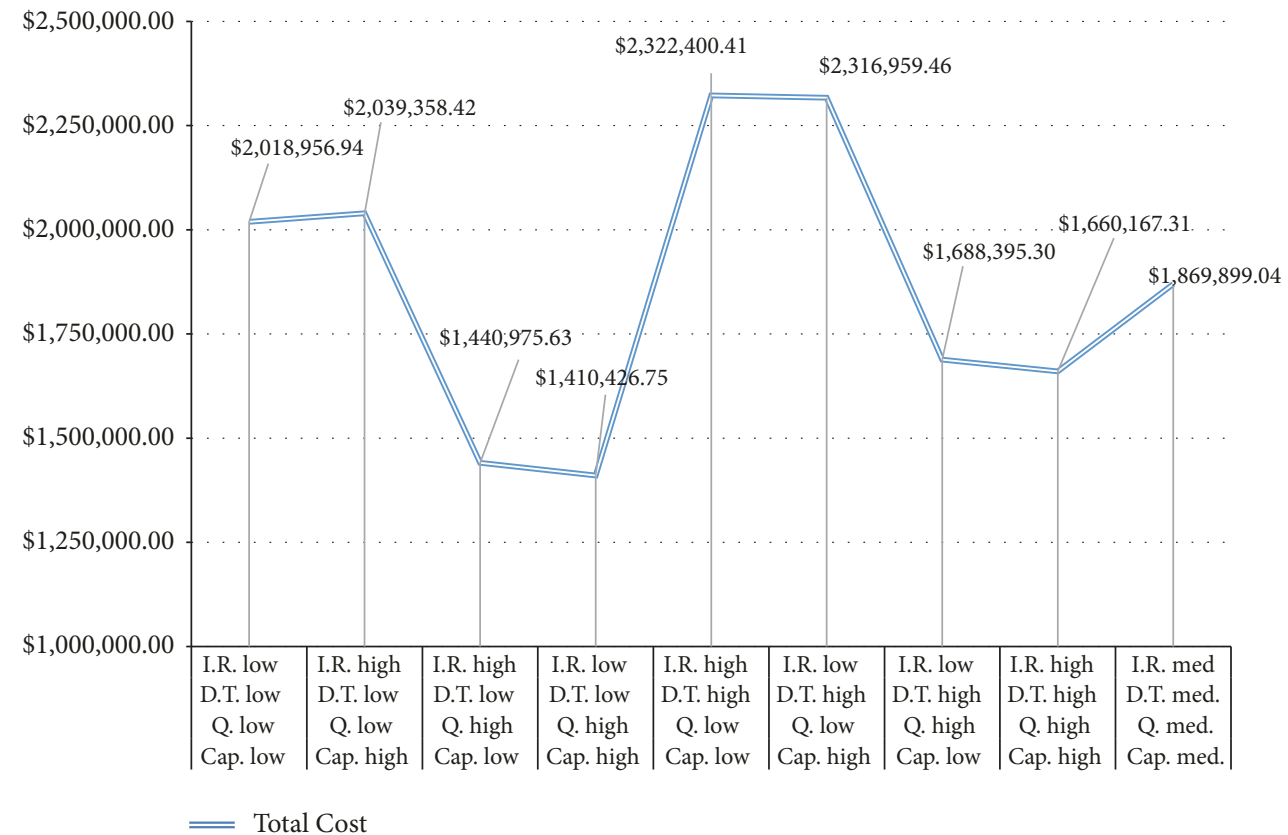

FIgURe 11: Total costs.

quality, followed by the effect of delivery time. Therefore, a policy is recommended in which, first, the variability of the quality in the supply chain is controlled and then care is taken regarding the variability produced by the delivery time. If it is possible to reduce transportation costs, higher profitability can be achieved. It can be observed that the factor that has less influence is the financing caused by the interest rate.

Results obtained in this paper are conditioned to the specific instance considered, with the specific parameters assumed. Thus, the yielded managerial insights cannot be easily extended to any other case, which may face different cost interactions. In each treatment, the level of each factor is set for the entire applicable scope in the supply chain, but with differences in parameters for different actors within the same echelon. In this way, the MILP optimizer drives the flow to laggard performers sometimes to keep up with the constraints. However, the pattern (laggard and best-inclass actors) between replicas, for the level change, is not conserved but is done randomly. This impedes a deeper analysis for particular cases, although the original scope 


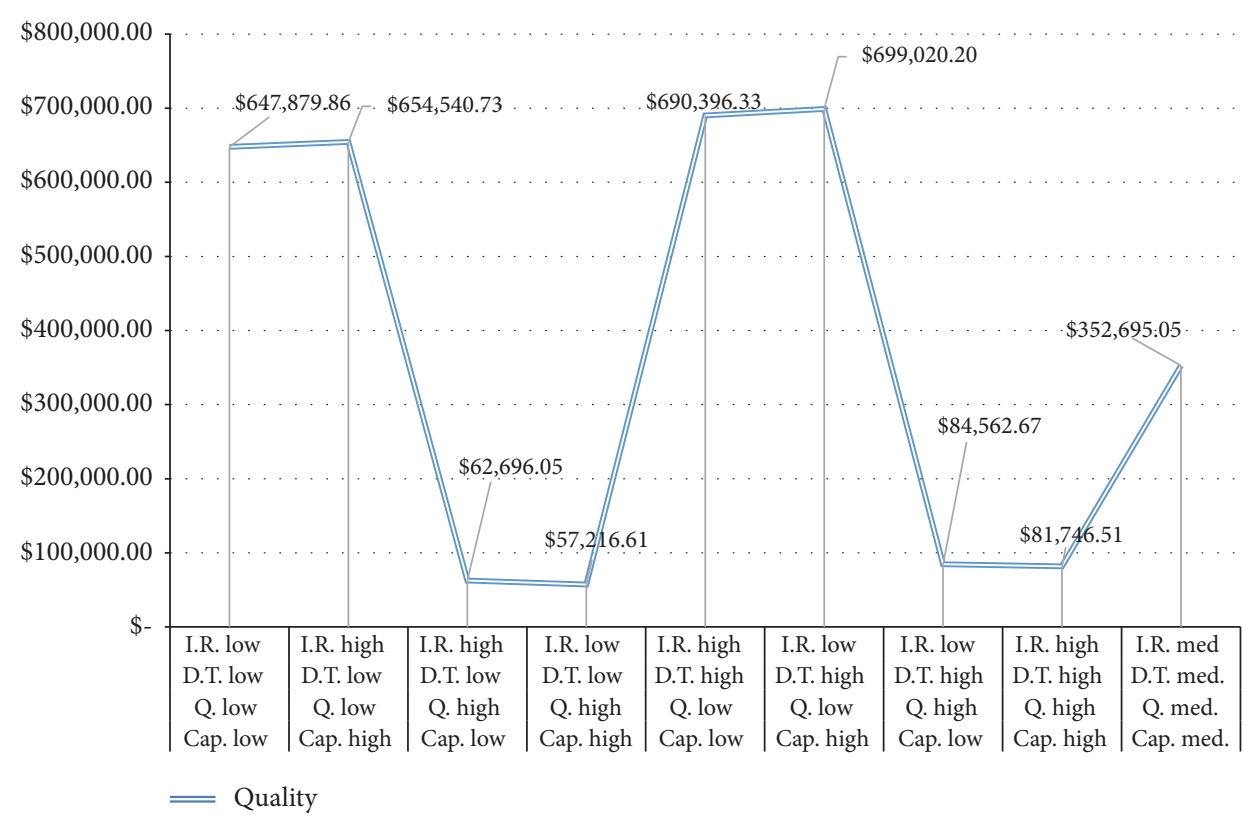

FIgURE 12: Quality cost.

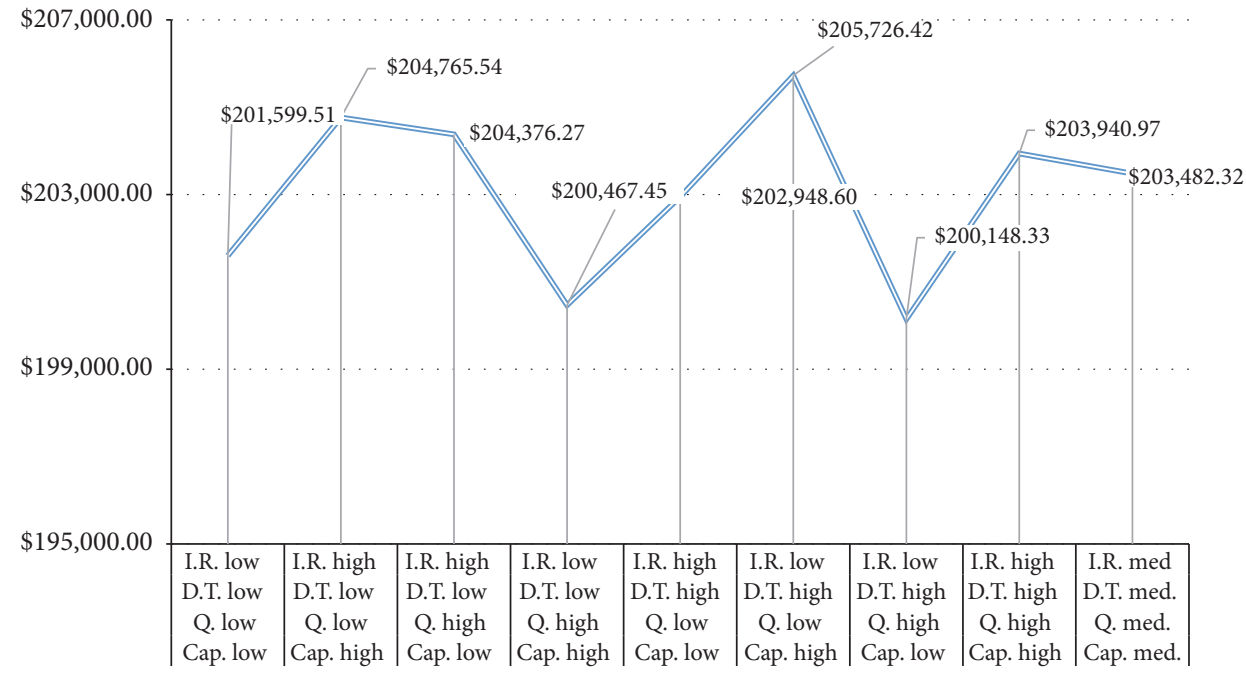

FIgURE 13: Raw material cost.

of the experiments aimed to know in a general way the effect of the factors on the organization and the performance (profit) of the supply chain. This is one key reason why the conclusions are not completely generalizable.

A main contribution of this paper stands on the methodological tool to analyze and optimize the supply chain network based on design of experiments and optimization techniques. As discussed, the proposed methodology can be easily extended to other case studies and problems, with different nature and features.

The application of design of experiments can be an alternative for stochastic programming, when the variability of parameters can be approximated by known probability distributions. The analysis allows measuring the effects of variability in the objective function, from main factors and interactions. A certain variability can be considered relatively high with respect to its own mean, but the effect can be minimal in the objective function, and this can be clarified by an analysis as proposed in this work.

Traditional methodologies on supply chain network literature are mainly focused on logistics cost minimization. In contrast, this research shows that quality costs may be very relevant and may present a great influence on system profit and supply chain network design and operation.

Several aspects can be considered as future research, to extend the applicability of this model. The most important are mentioned as follows: (i) it is intended to analyze the behavior of the supply chain by integrating learning curves for the 


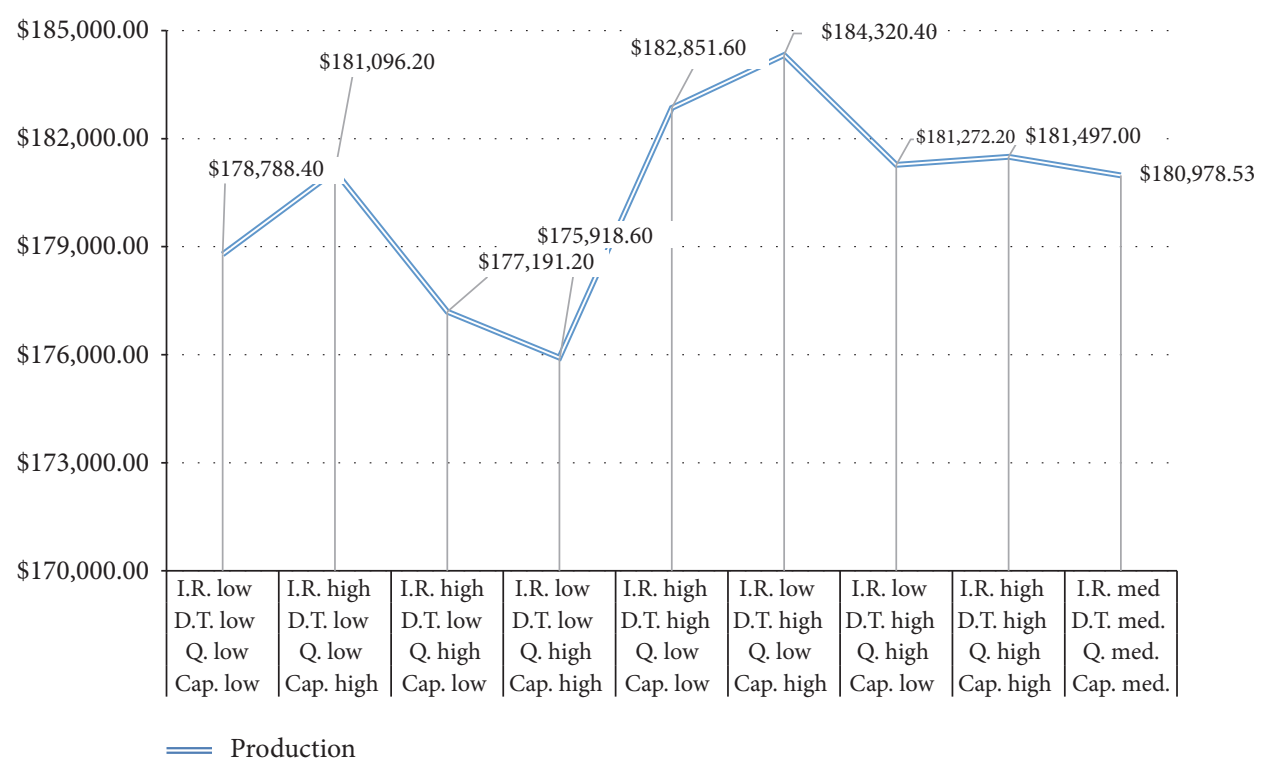

Figure 14: Production costs.

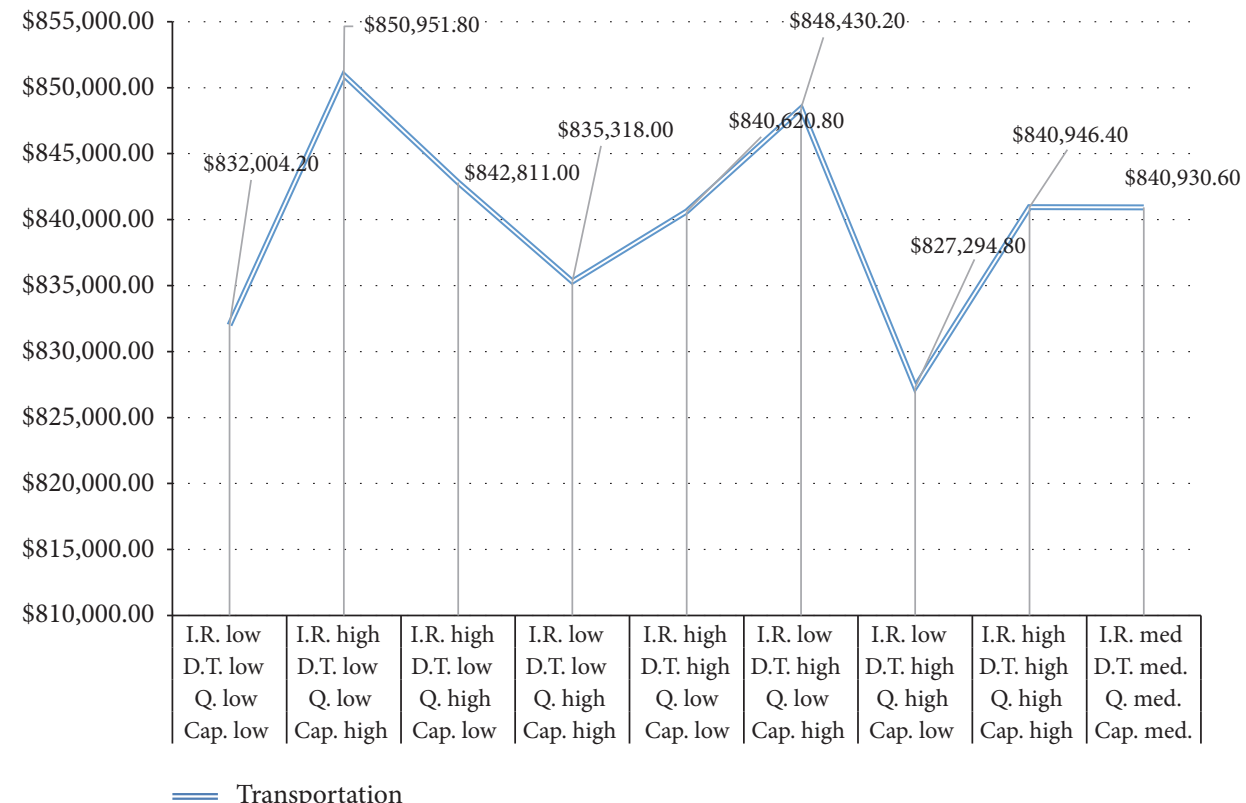

FIGURE 15: Transportation costs.

echelons, which may show the impact and the time it will take to incorporate a new participant in the supply chain. This can be applied when a supplier decides to enter into a different type of industry, in which it will have to go through a learning process to know the requirements of the new market. (ii) In this work, the quantities circulating through the supply chain have been considered as continuous variables. Typically, anyhow, many raw materials in most supply chains are handled in continuous measurement units, as liquids, chemicals, materials, and so on. It could be possible if necessary to transform this MILP to a full integer problem, considering its implications for yielding solutions, that may require the use of heuristics. (iii) For a different future case study, it is important to consider several costs that here were omitted, as other components of COPQ, like scrap costs. The same may be considered for the inventory holding costs and others. (iv) Stochastic demand and the implications derived from out-ofstock situations can be also considered in future analysis.

\section{Disclosure}

Pablo A. Miranda is a Visiting Researcher at Portsmouth Business School, University of Portsmouth, Portsmouth, UK. 


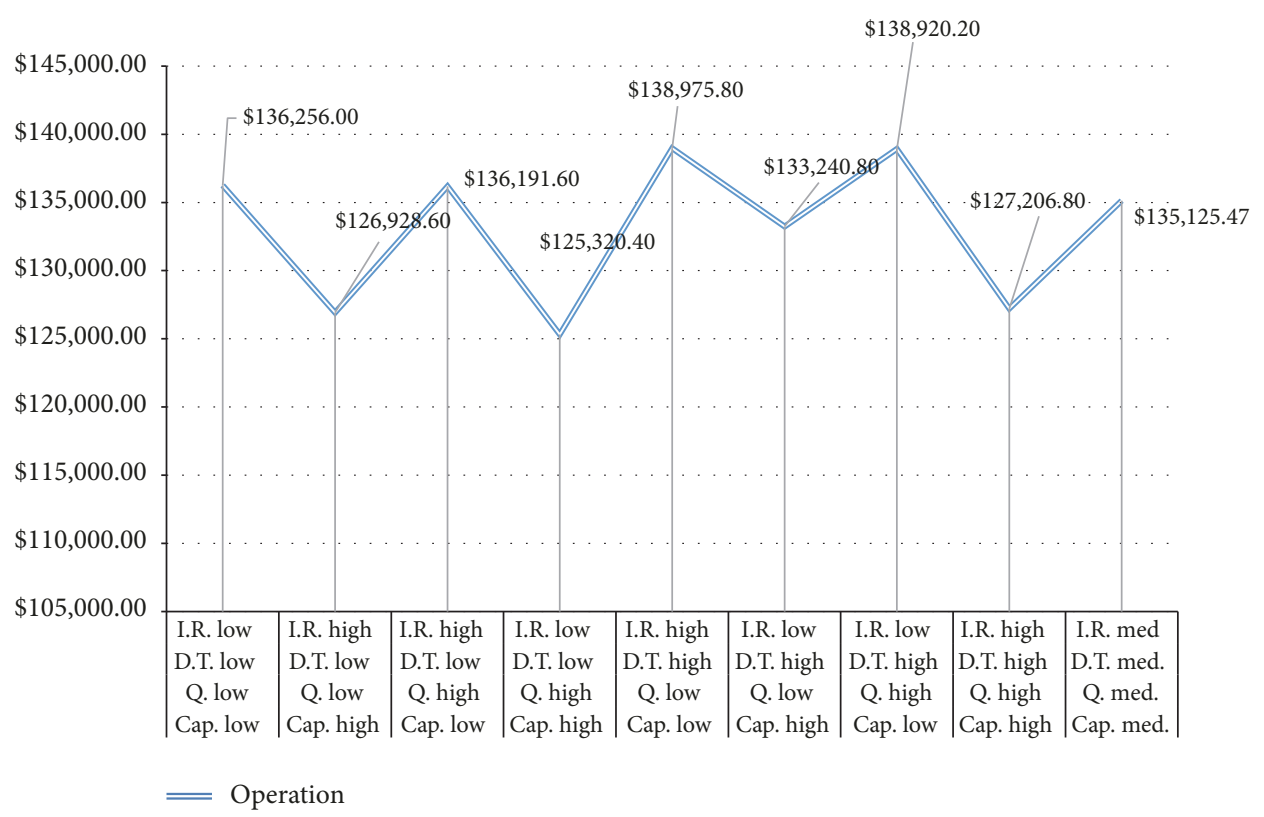

Figure 16: Operating costs.

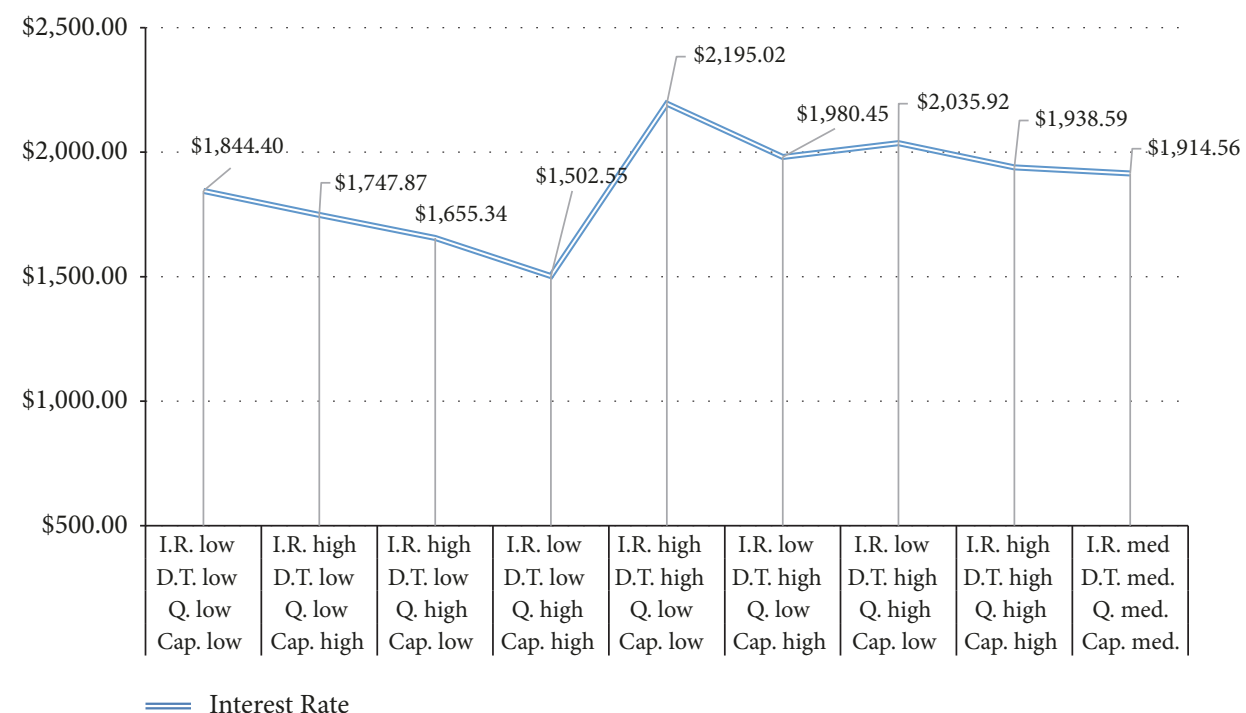

FIGURE 17: Interest rate (financial) costs.

\section{Conflicts of Interest}

The authors declare that they have no conflicts of interest.

\section{Acknowledgments}

This research has been partially funded by Universidad Panamericana through the grant "Fomento a la Investigación UP 2017," under Project Code UP-CI-2017-ING-GDL-07, and by the Universidad Técnica Federico Santa María through the grant "Proyectos de Investigación Internos 2017."

\section{References}

[1] Á. Bányai, T. Bányai, B. Illés, Á. Bányai, T. Bányai, and B. Illés, "Optimization of Consignment-Store-Based Supply Chain with Black Hole Algorithm," Complexity, 2017.

[2] R. E. Perez Loaiza, E. Olivares-Benitez, P. A. Miranda Gonzalez, A. Guerrero Campanur, and J. L. Martinez Flores, "Supply chain network design with efficiency, location, and inventory policy using a multiobjective evolutionary algorithm," International Transactions in Operational Research, vol. 24, no. 1-2, pp. 251275, 2017.

[3] K. K. Castillo-Villar and N. R. Smith, "Supply chain design including quality considerations: Modeling and solution 
approaches based on metaheuristics," Handbook of Research on Novel Soft Computing Intelligent Algorithms: Theory and Practical Applications, vol. 1-2, pp. 102-137, 2013.

[4] M. Bachlaus, M. K. Tiwari, and F. T. S. Chan, "Multi-objective resource assignment problem in a product-driven supply chain using a Taguchi-based DNA algorithm," International Journal of Production Research, vol. 47, no. 9, pp. 2345-2371, 2009.

[5] B. Raa, "Fleet optimization for cyclic inventory routing problems," International Journal of Production Economics, vol. 160, pp. 172-181, 2015.

[6] H. Hu, Y. Zhang, and L. Zhen, "A two-stage decomposition method on fresh product distribution problem," International Journal of Production Research, vol. 55, no. 16, pp. 4729-4752, 2017.

[7] W. Zhang and A. W. Xu, "Simulation-based robust optimization for the schedule of single-direction bus transit route: The design of experiment," Transportation Research Part E: Logistics and Transportation Review, vol. 106, no. Part E, pp. 203-230, 2017.

[8] R. Bucki and P. Suchánek, "Modelling decision-making processes in the management support of the manufacturing element in the logistic supply chain," Complexity, vol. 2017, Article ID 5286135, 15 pages, 2017.

[9] C.-C. Hsu, K. C. Tan, V. R. Kannan, and G. Keong Leong, "Supply chain management practices as a mediator of the relationship between operations capability and firm performance," International Journal of Production Research, vol. 47, no. 3, pp. 835-855, 2009.

[10] R. B. Franca, E. C. Jones, C. N. Richards, and J. P. Carlson, "Multi-objective stochastic supply chain modeling to evaluate tradeoffs between profit and quality," International Journal of Production Economics, vol. 127, no. 2, pp. 292-299, 2010.

[11] A. A. Javid and P. Hoseinpour, "A model for quality management in a supply chain with a retailer and a manufacturer," South African Journal of Industrial Engineering, vol. 21, no. 1, pp. 103111, 2010.

[12] K. Das, "A quality integrated strategic level global supply chain model," International Journal of Production Research, vol. 49, no. 1, pp. 5-31, 2011.

[13] B. Elahi, Y. Pakzad-Jafarabadi, L. Etaati, and S. M. Seyedhosseini, "Optimization of supply chain planning with considering defective rates of products in each echelon," Technology and Investment, vol. 2, no. 3, p. 211, 2011.

[14] B. M. Fathollah, M. R. Barzoki, and S. R. Hejazi, "A joint lotsizing and marketing model with reworks, scraps and imperfect products," International Journal of Industrial Engineering Computations, vol. 2, no. 2, pp. 395-408, 2011.

[15] K. K. Castillo-Villar, N. R. Smith, and J. L. Simonton, "A model for supply chain design considering the cost of quality," Applied Mathematical Modelling, vol. 36, no. 12, pp. 5920-5935, 2012.

[16] J. Chen, L. Liang, D. Yao, and S. Sun, "Price and quality decisions in dual-channel supply chains," European Journal of Operational Research, vol. 259, no. 3, pp. 935-948, 2017.

[17] S. Wang, Q. Hu, and W. Liu, "Price and quality-based competition and channel structure with consumer loyalty," European Journal of Operational Research, vol. 262, no. 2, pp. 563-574, 2017.

[18] J. Quigley, L. Walls, G. Demirel, B. L. MacCarthy, and M. Parsa, "Supplier quality improvement: The value of information under uncertainty," European Journal of Operational Research, vol. 264, no. 3, pp. 932-947, 2018.
[19] M. Yazdani, P. Zarate, A. Coulibaly, and E. K. Zavadskas, "A group decision making support system in logistics and supply chain management," Expert Systems with Applications, vol. 88, pp. 376-392, 2017.

[20] M. V. Asokan and V. K. G. Unnithan, "Estimation of vendor's process capability from the lots screened to meet specifications," Quality Engineering, vol. 11, no. 4, pp. 537-540, 1999.

[21] L. K. Chan, S. W. Cheng, and F. A. Spiring, "A multivariate measure of process capability," International Journal of Modelling \& Simulation, vol. 11, no. 1, pp. 1-6, 1991.

[22] V. E. Kane, "Process capability indices," Journal of Quality Technology, vol. 18, no. 1, pp. 41-52, 1986.

[23] N. L. Johnson and S. Kotz, "Process capability indices - a review, 1992-2000," Journal of Quality Technology, vol. 34, no. 1, pp. 2-19, 2002.

[24] W. L. Pearn and S. Kotz, Encyclopedia and Handbook of Process Capability Indices: A Comprehensive Exposition of Quality Control Measures (Series on Quality, Reliability and Engineering Statistics), World Scientific Publishing Co., 2006.

[25] H. K. Alfares and A. M. Attia, "A supply chain model with vendor-managed inventory, consignment, and quality inspection errors," International Journal of Production Research, vol. 55, no. 19, pp. 5706-5727, 2017.

[26] J. M. Juran, “Quality Trilogy," Quality Progress, vol. 19, no. 8, pp. 19-24, 1986.

[27] H. J. Harrington, "Poor-Quality Cost," 1987.

[28] S. C. Singhal, "Multiprocess performance analysis chart (MPPAC) with capability zones," Quality Engineering, vol. 4, no. 1, pp. 75-81, 1991.

[29] Y.-M. Chou, "Selecting a better supplier by testing process capability indices," Quality Engineering, vol. 6, no. 3, pp. 427438, 1994.

[30] R. J. Linn, F. Tsung, and L. W. C. Ellis, "Supplier selection based on process capability and price analysis," Quality Engineering, vol. 18, no. 2, pp. 123-129, 2006.

[31] C.-Y. Shen and K.-T. Yu, "An integrated fuzzy strategic supplier selection approach for considering the supplier integration spectrum," International Journal of Production Research, vol. 50, no. 3, pp. 817-829, 2012.

[32] P. Lundkvist, K. Vnnman, and M. Kulahci, "A comparison of decision methods for C pk when data are autocorrelated," Quality Engineering, vol. 24, no. 4, pp. 460-472, 2012.

[33] S. Bera and I. Mukherjee, "An integrated approach based on principal component and multivariate process capability for simultaneous optimization of location and dispersion for correlated multiple response problems," Quality Engineering, vol. 25, no. 3, pp. 266-281, 2013.

[34] M.-H. Shu and H.-C. Wu, "Supplier evaluation and selection based on stochastic dominance: A quality-based approach," Communications in Statistics-Theory and Methods, vol. 43, no. 14, pp. 2907-2922, 2014.

[35] X. Tang and A. Rai, "The moderating effects of supplier portfolio characteristics on the competitive performance impacts of supplier-facing process capabilities," Journal of Operations Management, vol. 30, no. 1-2, pp. 85-98, 2012.

[36] B. M. Hsu, L. Y. Hsu, and M. H. Shu, "Evaluation of supply chain performance using delivery-time performance analysis chart approach," Journal of Statistics and Management Systems, vol. 16, no. 1, pp. 73-87, 2013.

[37] M. A. Bushuev, "Delivery performance improvement in twostage supply chain," International Journal of Production Economics, vol. 195, pp. 66-73, 2018. 
[38] H. Fang and R. Xiao, "Cycle quality chain early warning network with e-channel lead time disruption," International Journal of Systems Science: Operations \& Logistics, vol. 1, no. 1, pp. 47-67, 2014.

[39] Y. Zhou, B. Cao, Y. Zhong, and Y. Wu, "Optimal advertising/ordering policy and finance mode selection for a capitalconstrained retailer with stochastic demand," Journal of the Operational Research Society, vol. 68, no. 12, pp. 1620-1632, 2017.

[40] S. R. Hejazi, J. C. Tsou, and M. R. Barzoki, "Optimal lot size of EPQ model considering imperfect and defective products," Journal of Industrial Engineering International, vol. 4, no. 7, pp. 59-68, 2008.

[41] The European Chemical Industry Council CEFIC, Good Manufacturing Practices for Active Ingredient Manufacturers, European Federation of Pharmaceutical Industries, 1996.

[42] S. Kumar, M. L. McCreary, and D. A. Nottestad, "Quantifying supply chain trade-offs using six sigma, simulation, and designed experiments to develop a flexible distribution network," Quality Engineering, vol. 23, no. 2, pp. 180-203, 2011.

[43] A. Madadi, M. E. Kurz, S. J. Mason, and K. M. Taaffe, "Supply chain design under quality disruptions and tainted materials delivery," Transportation Research Part E: Logistics and Transportation Review, vol. 67, pp. 105-123, 2014. 


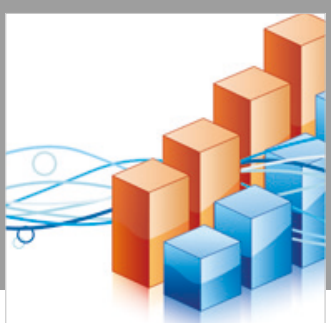

Advances in

Operations Research

\section{-n-m}
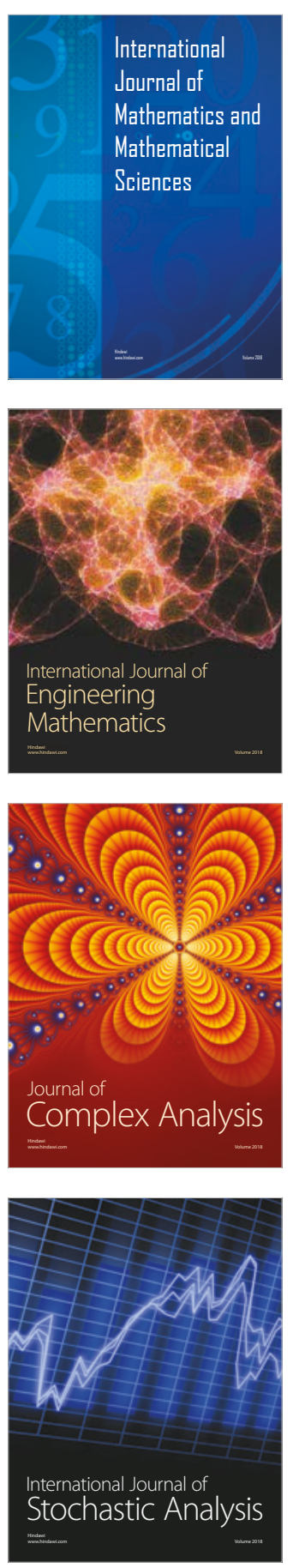
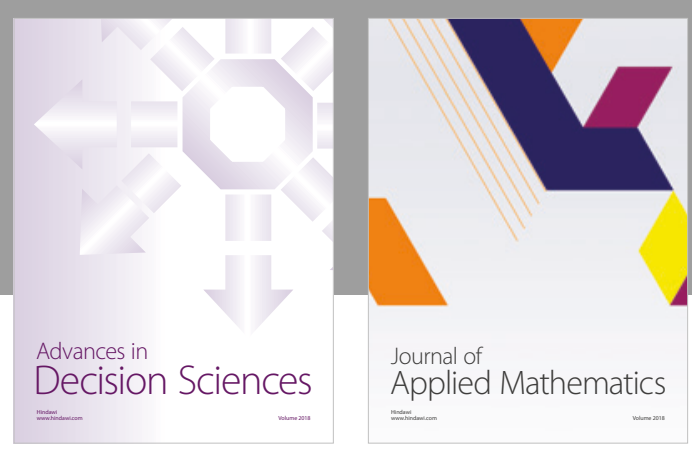

Journal of

Applied Mathematics
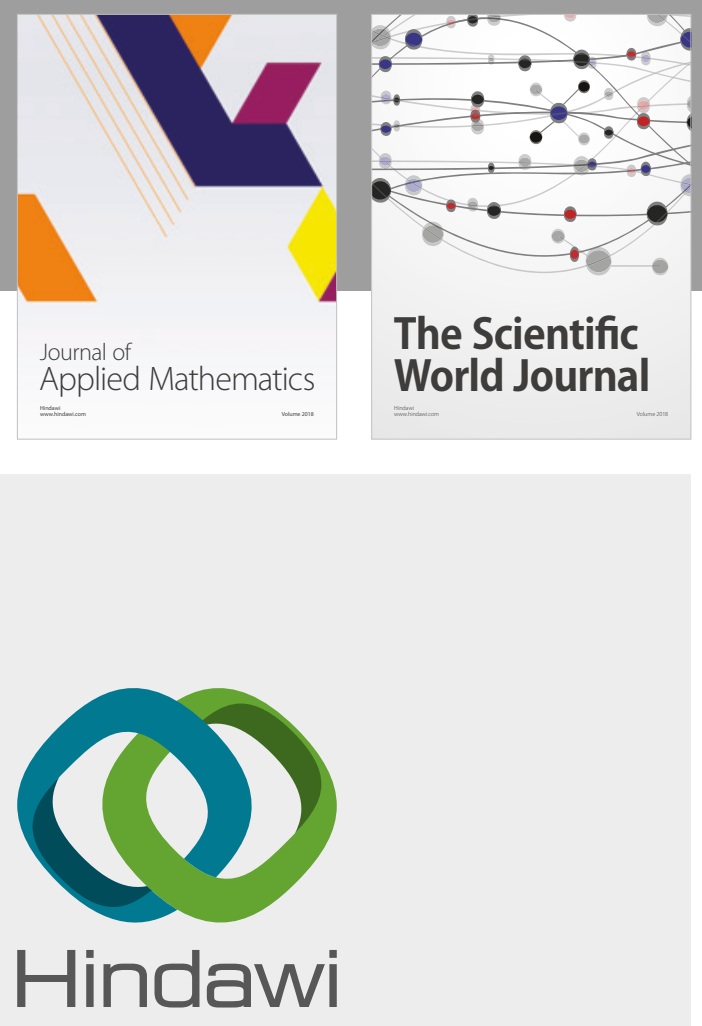

Submit your manuscripts at

www.hindawi.com

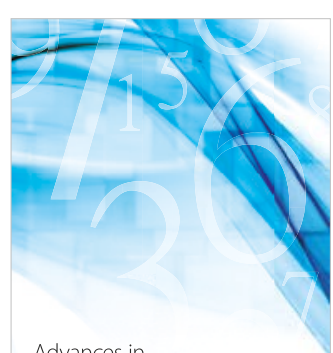

Advances in
Numerical Analysis
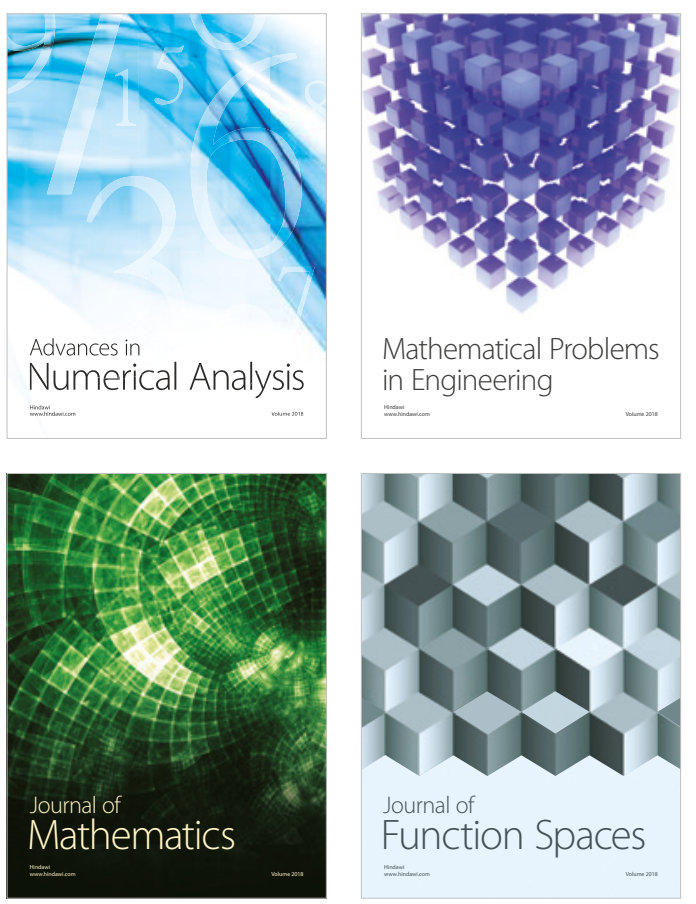

Mathematical Problems in Engineering

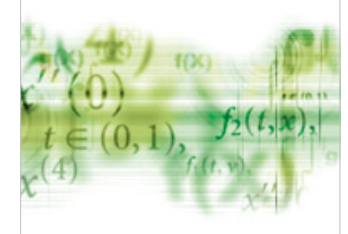

International Journal of

Differential Equations

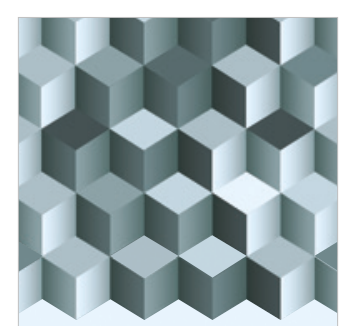

Journal of

Function Spaces
The Scientific

World Journal

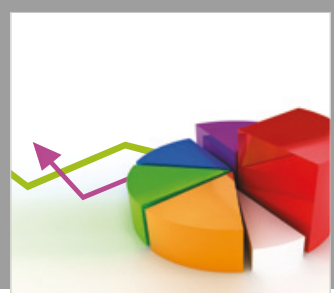

Journal of

Probability and Statistics
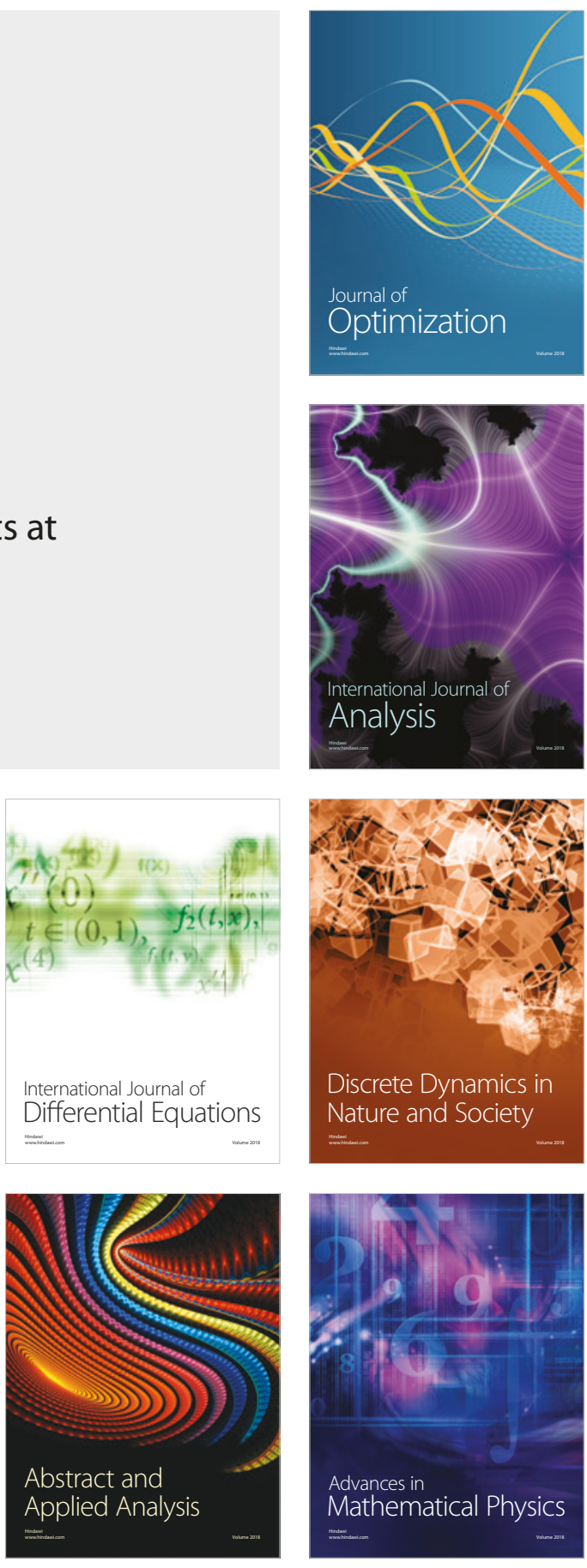